\title{
CCD imaging of twenty nearby isolated irregular galaxies ${ }^{\star}$
}

\author{
L.N. Makarova and I.D. Karachentsev
}

Special Astrophysical Observatory, Russian Academy of Sciences, N. Arkhyz, KChR, Stavropolsky kraj 357147, Russia

Received April 7; accepted April 20, 1998

\begin{abstract}
We present $B, V$ and $I$ CCD stellar photometry for a sample of 20 field irregular dwarf galaxies. Their corrected radial velocity is $V_{0}<500 \mathrm{~km} \mathrm{~s}^{-1}$. Most of them have been resolved into stars for the first time. Based on photometry of their brightest blue stars we have derived the following distances: 5.9 Mpc (UGC 685), 5.4 Mpc (UGC 1281), 7.2 Mpc (UGC 3303), 7.0 Mpc (UGC 3476), 7.3: Mpc (UGC 3600), 7.2: Mpc (UGC 3698), 7.9 Mpc (NGC 2337), 8.6 Mpc (UGC 3817), 5.7 Mpc (UGC 3860), $5.6 \mathrm{Mpc}$ (UGC 4426), $\geq 7.9 \mathrm{Mpc}$ (F 565-v1), 7.4: Mpc (UGC 5086), 7.1 Mpc (UGC 5272), 5.9 Mpc (UGC 5340), 7.1 Mpc (UGC 5427), 2.7: Mpc (UGC 5456), 6.6 Mpc (NGC 3274), 9.3 Mpc (UGC 5889), 5.2 Mpc (NGC 5238), and 8.0 Mpc (UGC 9405).

Our sample exhibits diverse morphological properties evidently caused by their different starburst activity. The galaxy sample has a median integral absolute magnitude $M_{B}=-14.6$ and a median integral colour $(B-V)_{\mathrm{T}}=$ +0.47 . One dwarf, UGC 5340 , stands out by its very blue colour, $(B-V)_{\mathrm{T}}=+0.18$, and by its high $M(\mathrm{HI}) / L$ ratio, as expected for young galaxies. Four objects of the sample are IRAS sources.

Being well isolated systems, the considered galaxies may be used to estimate a local value of the Hubble parameter, $H=V_{0} / D$. For half of the sample galaxies their individual $\mathrm{H}$ - values are concentrated within [58-68] $\mathrm{km} \mathrm{s}^{-1} / \mathrm{Mpc}$ with a median of $65 \mathrm{~km} \mathrm{~s}^{-1} / \mathrm{Mpc}$.
\end{abstract}

Key words: galaxies: distances - galaxies: stellar content — galaxies: dwarf; irregular

\section{Introduction}

This paper continues a series of publications (Karachentsev et al. 1997; Makarova et al. 1998;

\footnotetext{
* Tables 2-17 are only available in electronic form at the CDS via anonymous ftp to cdsarc.u-strasbg.fr (130.79.128.5) or via http://cdsweb.u-strasbg.fr/Abstract.html
}

Karachentsev \& Drozdowsky 1998) devoted to photometry of the brightest stars in nearby late-type galaxies, using the CCD camera of the 2.5 meter Nordic telescope. Together with results obtained at the 6 meter telescope, these data consist a part of our general program aimed at the measurement of photometric distances for a complete sample of the nearest galaxies with radial velocities within $500 \mathrm{~km} \mathrm{~s}^{-1}$. Nearing completion, the program will yield a representative atlas of detailed images for about 150 dwarf irregular galaxies. The availability of such a volume-limited "gallery" allows the structural properties of dwarf galaxies to be classified in connection with diverse conditions of star formation in high and low density regions of the Local universe.

Now we will consider 20 mainly isolated dwarf galaxies. Most of them have been resolved into stars for the first time.

\section{Observations and photometry}

All the galaxies were observed on February 4-7, 1995 at the 2.56-meter Nordic telescope (La Palma). A CCD camera with a TEC $1 \mathrm{k} \times 1 \mathrm{k}$ chip provided a field of view of $3 \times 3$ arcmin with a resolution of 0 "' 176 per pixel. The exposure times in filters of the $B, V, I$ Johnson-Cousins system as well as the seeing $(F W H M)$ and the air masses are presented in Table 1. The CCD frames were debiased, flatfielded and calibrated by the equatorial standards from Landolt (1992). Stellar photometry was carried out with the DAOPHOT package (Stetson 1987). For the analysis we selected, as a rule, starlike objects with standard photometric errors $\sigma(V) \leq 0.2 \mathrm{mag}$ and image parameters $|\mathrm{SHARP}| \leq 2.5$.

The CCD frames for the 20 observed galaxies in the $B, V$ or $I$ filters are presented in Figs. 1-20. Our stellar photometry results are given in Tables 2-17, which are accessible from the CDS via ftp. Besides the detailed photometry of starlike objects, we made a surface photometry 
Table 1. Observation log

\begin{tabular}{|c|c|c|c|c|c|}
\hline Name & Date & Filter & $\begin{array}{c}\text { Exposure } \\
\text { (sec) }\end{array}$ & $\begin{array}{l}\text { FWHM } \\
(\operatorname{arcsec})\end{array}$ & Airmass \\
\hline \multirow[t]{2}{*}{ UGC 685} & 7.02 .95 & V & 600 & 1.1 & 1.56 \\
\hline & & $I$ & 600 & 1.0 & 1.56 \\
\hline \multirow[t]{2}{*}{ UGC 1281} & 7.02 .95 & $V$ & 600 & 1.0 & 1.39 \\
\hline & & $I$ & 300 & 1.2 & 1.39 \\
\hline \multirow[t]{2}{*}{ UGC 3303} & 5.02 .95 & $V$ & 300 & 1.4 & 1.10 \\
\hline & & $I$ & 600 & 1.0 & 1.09 \\
\hline \multirow[t]{2}{*}{ UGC 3476} & 5.02 .95 & $V$ & 600 & 0.7 & 1.03 \\
\hline & & $I$ & 600 & 0.6 & 1.03 \\
\hline \multirow[t]{2}{*}{ UGC 3600} & 5.02 .95 & V & 300 & 1.4 & 1.04 \\
\hline & & $I$ & 300 & 1.3 & 1.04 \\
\hline \multirow[t]{2}{*}{ UGC 3698} & 5.02 .95 & V & 600 & 1.5 & 1.06 \\
\hline & & $I$ & 600 & 1.7 & 1.06 \\
\hline \multirow[t]{2}{*}{ NGC 2337} & 5.02 .95 & V & 600 & 1.9 & 1.04 \\
\hline & & $I$ & 600 & 1.7 & 1.04 \\
\hline \multirow[t]{2}{*}{ UGC 3817} & 7.02 .95 & V & 600 & 0.6 & 1.04 \\
\hline & & $I$ & 600 & 0.6 & 1.04 \\
\hline \multirow[t]{2}{*}{ UGC 3860} & 6.02 .95 & V & 600 & 0.7 & 1.03 \\
\hline & & $I$ & 600 & 0.7 & 1.03 \\
\hline \multirow[t]{2}{*}{ UGC 4426} & 7.02 .95 & $B$ & 600 & 1.3 & 1.13 \\
\hline & & $V$ & 600 & 1.0 & 1.13 \\
\hline \multirow[t]{2}{*}{ F 565-v1 } & 7.02 .95 & $B$ & 600 & 1.1 & 1.10 \\
\hline & & $V$ & 600 & 1.1 & 1.09 \\
\hline \multirow[t]{2}{*}{ UGC 5086} & 5.02 .95 & V & 600 & 1.8 & 1.01 \\
\hline & & $I$ & 600 & 1.6 & 1.01 \\
\hline \multirow[t]{2}{*}{ UGC 5272} & 7.02 .95 & $B$ & 300 & 1.1 & 1.12 \\
\hline & & $V$ & 300 & 0.9 & 1.12 \\
\hline \multirow[t]{2}{*}{ UGC 5340} & 7.02 .95 & $B$ & 300 & 1.0 & 1.18 \\
\hline & & $V$ & 300 & 0.8 & 1.18 \\
\hline \multirow[t]{2}{*}{ UGC 5427} & 7.02 .95 & $B$ & 300 & 1.0 & 1.07 \\
\hline & & $V$ & 300 & 1.0 & 1.07 \\
\hline \multirow[t]{2}{*}{ UGC 5456} & 7.02 .95 & $B$ & 300 & 1.3 & 1.09 \\
\hline & & $V$ & 300 & 1.1 & 1.09 \\
\hline \multirow[t]{2}{*}{ NGC 3274} & 6.02 .95 & $B$ & 600 & 1.5 & 1.05 \\
\hline & & $V$ & 600 & 1.2 & 1.07 \\
\hline \multirow[t]{2}{*}{ UGC 5889} & 7.02 .95 & $B$ & 300 & 0.9 & 1.17 \\
\hline & & $V$ & 300 & 0.8 & 1.17 \\
\hline \multirow[t]{2}{*}{ NGC 5238} & 7.02 .95 & $B$ & 200 & 1.0 & 1.09 \\
\hline & & $V$ & 100 & 1.1 & 1.09 \\
\hline \multirow[t]{2}{*}{ UGC 9405} & 7.02 .95 & $B$ & 500 & 0.8 & 1.13 \\
\hline & & $V$ & 500 & 0.8 & 1.13 \\
\hline
\end{tabular}

of the galaxies in concentric circular apertures, measuring their total apparent magnitudes, $V_{\mathrm{T}}$, and integral colour indices, $(B-V)_{\mathrm{T}}$ or $(V-I)_{\mathrm{T}}$. These data are listed in Table 18.

\section{Distance estimates}

The photometric limit of our CCD frames was about $V=23 \mathrm{mag}$, which allowed us to reveal only the brightest stars (supergiants) in the galaxies. Following Sandage \& Tammann (1974) and de Vaucouleurs (1978) we selected blue supergiant candidates under the condition $B-V<0.4$, and used the mean apparent magnitude of three brightest stars, $\langle B(3 B)>$, as a distance indica- tor to a galaxy. The distance moduli were estimated from the relation

$\mu_{0}(B)=1.51<B(3 B)>-0.51 B_{\mathrm{T}}-A_{B}+4.14$,

where $A_{B}$ is the extinction, while the numerical coefficients were calibrated (Karachentsev \& Tikhonov 1994) from galaxies with distances derived from cepheids.

It is well known that the probability for red supergiants to occur in a dwarf galaxy is rather low. In some cases when we found a suitable red supergiant candidate with an apparent magnitude $V(R 1)$ and a colour $(B-V)>1.6$, we estimated the distance modulus from the relation

$\mu_{0}(R 1)=1.10 V(R 1)-0.10 B_{\mathrm{T}}-0.76 A_{B}+7.00$,

which was also calibrated from galaxies with distances known from cepheids.

Some basic parameters of the galaxies as well the results of our photometry are presented in Table 18. Its columns contain: (1) - the galaxy name; $(2)$ - the standard diameter from the PGC (Paturel et al. 1992) in arcmin; (3), (4) - the total apparent magnitude and the integrated colour index from our measurements; (5) - the galactic extinction; (6), (7) - the heliocentric radial velocity from the PGC and the corrected velocity with respect to the Local Group centroid according to Karachentsev \& Makarov (1996); (8), (9) — the mean apparent magnitude and the mean colour index for the three brightest blue stars in the galaxy, used for its distance estimation; (10), (11) - the distance modulus and the corresponding distance in Mpc, (12) - the galaxy absolute magnitude.

In the cases where a galaxy was observed in the $V$ and $I$ bands, the $(B-V)$ colour was derived from the relation $\{(B-V) \propto(V-I)\}$ in Fig. 21. Here the dots correspond to the standard Landolt stars. The circles and crosses indicate integrated colours of 65 and 49 galaxies measured by Reshetnikov \& Combes (1996) and Heraudeau \& Simien (1996), respectively. In a linear approximation the colourcolour relation may be fitted by $(B-V)=k(V-I)$ with $k_{\mathrm{b}}=0.83$ and $k_{\mathrm{r}}=0.91$ for blue and red stars, respectively. The integrated colours of the galaxies follow the relation $(B-V)_{\mathrm{T}}=0.85(V-I)_{\mathrm{T}}-0.20$, shifted to the right of the standard stars. This agrees well with the spectrophotometric data of Fukugita et al. (1995).

Below we give some notes on the observed galaxies.

UGC $685=$ KIG 45. As a very isolated system, the galaxy is listed in the Catalogue of Karachentseva (1973). Within its apparent boundary there no red stars. Based on three brightest blue stars (\#11, 19 and 6 in Table 2) we estimated the galaxy distance modulus to be 28.89 mag.

UGC 1281 = FGC 195. This edge-on galaxy is listed in the Flat Galaxy Catalogue by Karachentsev et al. (1993). One arcmin to the East, there is a compact elliptical galaxy. UGC 1281 has a high colour index for a Sm, which may be caused by internal extinction in the galaxy. Within its apparent boundary there is a red star, \#18 in Table 3, with $V=21.23$ and $V-I=+1.93$. Identifying it with a red supergiant, we derive a distance modulus 
Table 18. Basic parameters and distance estimates for 20 dwarf galaxies

\begin{tabular}{|cccccccccccc|}
\hline Name & $\begin{array}{c}a_{25} \\
\left({ }^{\prime}\right)\end{array}$ & $\begin{array}{c}B \mathrm{~T} \\
(\mathrm{mag})\end{array}$ & $\begin{array}{c}B-V)_{\mathrm{T}} \\
(\mathrm{mag})\end{array}$ & $\begin{array}{c}A_{B} \\
(\mathrm{mag})\end{array}$ & $\begin{array}{c}V_{\mathrm{h}} \\
\left(\mathrm{km} \mathrm{s}^{-1}\right)\end{array}$ & $\begin{array}{c}V_{0} \\
\left(\mathrm{~km} \mathrm{~s}^{-1}\right)\end{array}$ & $\begin{array}{c}\langle B(3 B)\rangle \\
(\mathrm{mag})\end{array}$ & $\begin{array}{c}<B-V\rangle_{3} \\
(\mathrm{mag})\end{array}$ & $\begin{array}{c}\mu_{0}(3 B) \\
(\mathrm{mag})\end{array}$ & $\begin{array}{c}D \\
\mathrm{Mpc}\end{array}$ & $\begin{array}{c}M_{B} \\
(\mathrm{mag})\end{array}$ \\
\hline UGC 685 & 1.4 & 14.44 & 0.54 & 0.11 & +155 & +349 & 21.33 & +0.18 & 28.89 & 5.95 & -14.56 \\
UGC 1281 & 4.5 & 13.26 & 0.71 & 0.15 & +157 & +367 & 20.83 & +0.15 & 28.68 & 5.44 & -15.67 \\
UGC 3303 & 3.6 & $13.0:$ & - & 0.58 & +521 & +447 & 21.42 & +0.12 & 29.27 & 7.16 & -16.85 \\
UGC 3476 & 1.0 & 15.38 & 0.73 & 0.98 & +469 & +477 & 22.45 & +0.20 & 29.23 & 7.01 & -14.83 \\
UGC 3600 & 1.1 & 15.58 & 0.58 & 0.39 & +403 & +426 & $22.2:$ & - & $29.3:$ & $7.3:$ & $-14.1:$ \\
& & & & & & & & & & & \\
UGC 3698 & 1.0 & 15.16 & 0.56 & 0.38 & +423 & +466 & $22.0:$ & - & $29.3:$ & $7.2:$ & $-14.5:$ \\
NGC 2337 & 2.0 & 13.10 & 0.57 & 0.38 & +434 & +477 & 21.46 & +0.29 & 29.48 & 7.88 & -16.76 \\
UGC 3817 & 1.8 & 15.96 & 0.71 & 0.36 & +438 & +479 & 22.53 & +0.07 & 29.66 & 8.55 & -14.06 \\
UGC 3860 & 1.3 & 14.96 & 0.41 & 0.23 & +354 & +371 & 21.53 & +0.13 & 28.79 & 5.73 & -14.06 \\
UGC 4426 & 2.0 & 15.27 & 0.45 & 0.13 & +394 & +399 & 22.78 & -0.03 & $30.62:$ & $5.6 \mathrm{R}$ & $-13.6:$ \\
& & & & & & & & & & & \\
F 565-v1 & 0.7 & 16.95 & 0.47 & 0.01 & +491 & +379 & $22.5:$ & - & $29.5:$ & $7.9:$ & $-12.5:$ \\
UGC 5086 & 1.0 & 15.94 & 0.71 & 0.07 & $+543:$ & - & - & - & {$[29.36]$} & {$[7.45]$} & -13.49 \\
UGC 5272 & 2.0 & 14.45 & 0.31 & 0.04 & +520 & +460 & 21.54 & 0.00 & 29.26 & 7.10 & -14.85 \\
UGC 5340 & 2.5 & 14.60 & 0.18 & 0.03 & +502 & +428 & 21.32 & -0.10 & 28.85 & 5.89 & -14.28 \\
UGC 5427 & 1.2 & 14.89 & 0.44 & 0.00 & +495 & +424 & 21.67 & +0.08 & 29.26 & 7.14 & -14.37 \\
UGC 5456 & 1.6 & 13.84 & 0.42 & 0.00 & +535 & +368 & 19.94 & +0.07 & $27.19:$ & $2.74:$ & $-13.4:$ \\
NGC 3274 & 2.1 & 13.25 & 0.40 & 0.06 & +537 & +461 & 21.03 & +0.03 & 29.08 & 6.55 & -15.89 \\
UGC 5889 & 1.8 & 14.25 & 0.60 & 0.04 & +572 & +431 & 21.87 & +0.12 & 29.85 & 9.33 & -15.64 \\
NGC 5238 & 1.3 & 13.80 & 0.40 & 0.00 & +233 & +346 & 20.84 & -0.04 & 28.57 & 5.18 & -14.77 \\
UGC 9405 & 1.9 & 14.65 & 0.57 & 0.00 & +221 & +384 & 22.00 & +0.01 & $<29.52>8.02:$ & $-14.9:$ \\
\hline
\end{tabular}

$\mu_{0}(R 1)=28.91$, which agrees well with a modulus of 28.68 from three brightest blue stars.

UGC 3303. A very bright red star is projected onto the central part of the galaxy, which shows some signs of spiral structure. An accurate photometry of the galaxy is not possible. We can find only a few stars in its images. Derived from three brightest blue of them (\#27, 40, and 44 in Table 4) the galaxy distance modulus is 29.27. As mentioned by Karachentsev \& Musella (1996), UGC 3303, together with the irregular dwarfs PGC 17716 and A0554+07, may belong to the scattered "Orion" group in the Milky Way zone of avoidance.

UGC 3476. This galaxy without central condensation looks like a footprint. On the I frame, within the galaxy boundary there are about 50 faint stars and compact knots. The brightest blue object (\#89 in Table 5) with $V=21.35$ and $V-I=-0.30$ is probably not a single star. The next three brightest blue supergiant candidates give a distance modulus of $29.23 \mathrm{mag}$.

UGC 3600. This is a very isolated irregular galaxy. On the frames with a $F W H M=1$ ". 4 we can distinguish only several bluish starlike objects. Their mean magnitude $<B(3 B)>\simeq 22.2$ gives a rough distance modulus estimate of 29.3 .

UGC 3698. UGC 3698 was also observed with a poor seeing. Its distance modulus estimate via the brightest blue objects, $\mu(3 B)=29.3$, also needs to be confirmed.

NGC $2337=$ UGC $3711=$ IRAS 07066+4432. The galaxy has a significant brightness gradient. Its central region is shown separately on the insert of Fig. 7. Several blue compact knots are situated around its bright nucleus. In the outer parts of the galaxy one can see a faint ring-like polar feature. Being an IRAS source, the galaxy apparently has a large amount of dust. From the brightest blue starlike objects (\#22, 24 and 29 in Table 6) its distance modulus is 29.48. Note that NGC 2337 and UGC 3698 are separated by 10 arcmin and have a radial velocity difference of $11 \mathrm{~km} \mathrm{~s}^{-1}$ only. Their similar distances favour the idea of gravitational binding of these irregular systems.

UGC 3817. This is a low surface brigtness galaxy without central condensation. All its brightest blue stars are fainter than $V=22 \mathrm{mag}$. Via three of them (\#40, 58, and 38 in Table 7) the galaxy distance modulus is 29.66 . Within the galaxy boundary there is one red star, \#37, with $V=21.62$ and $V-I=+2.28$. Being a probable red supergiant, it gives a modulus $\mu_{0}(R 1)=28.91$. Judging by the smoothed profile of UGC 3817 and its yellowish integral colour, $(B-V)_{\mathrm{T}}=+0.62$, the galaxy may be a dwarf evolved system having lost massive blue stars. In such a case the distance obtained from the brightest blue stars may be overestimated.

UGC $3860=$ KIG $190=$ DDO 43. Georgiev et al. (1997) have already resolved it into stars. UGC 3860 has no prominent blue complexes, except for one compact object, \#39, with $V=21.34$ and $V-I=-0.41$ at the NE-edge of the galaxy. Three blue supergiant candidates (\#41, 33 and 22 in Table 8) yield a distance modulus of 28.79 , which is lower than the estimate, 29.23, given by Georgiev et al. (1997). 


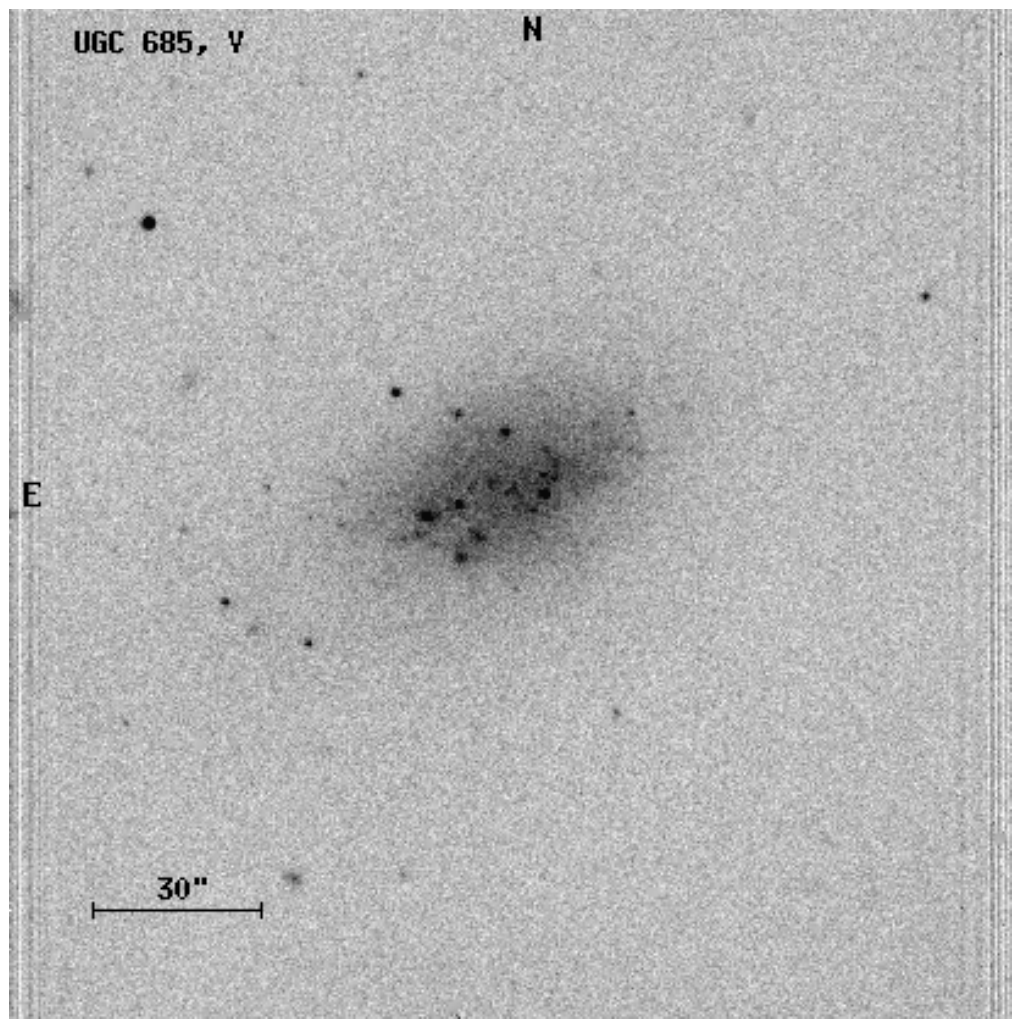

Fig. 1. The $V$ frame of UGC 685. As for all other images, North is top, and East is left. The upper right corner corresponds to $X=0, Y=0$ of the frame coordinates given in the tables; $X$ grows to East and $Y$ to South

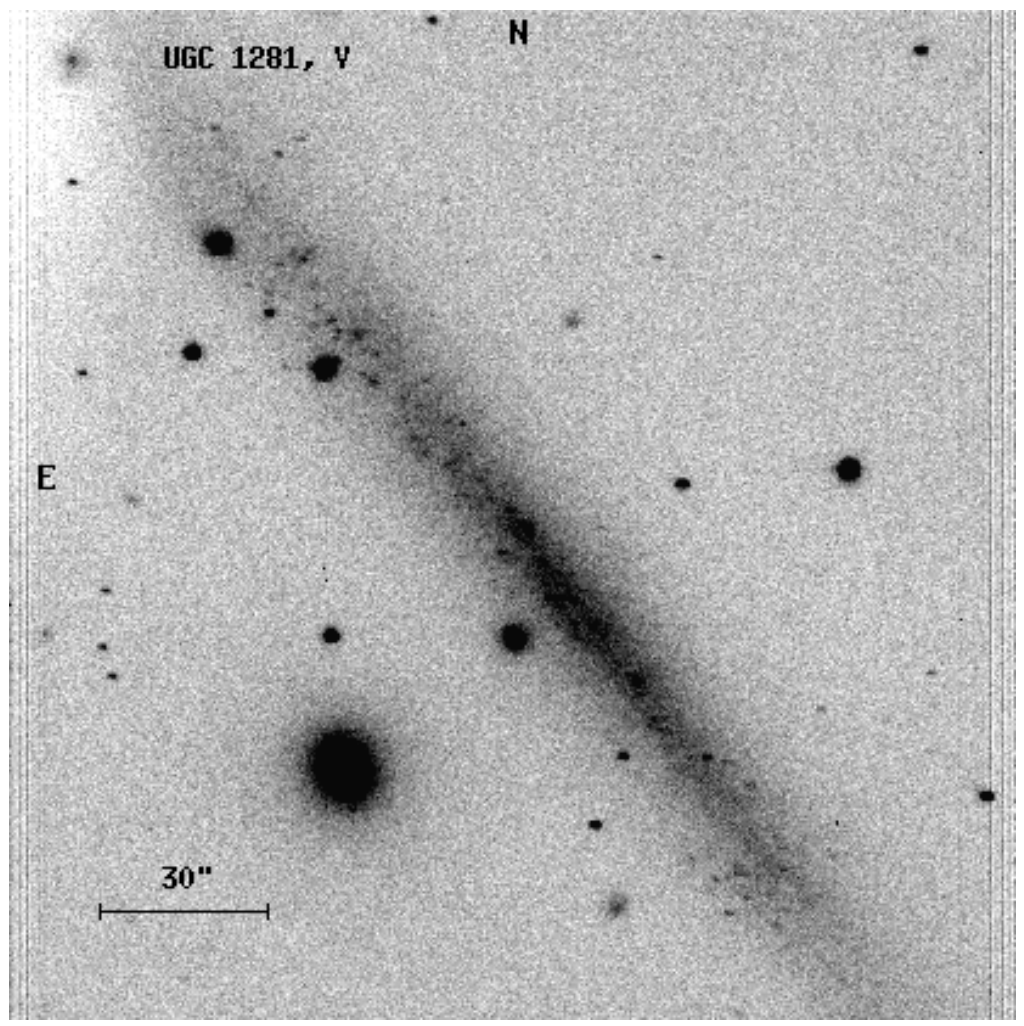

Fig. 2. The $V$ frame of UGC 1281 


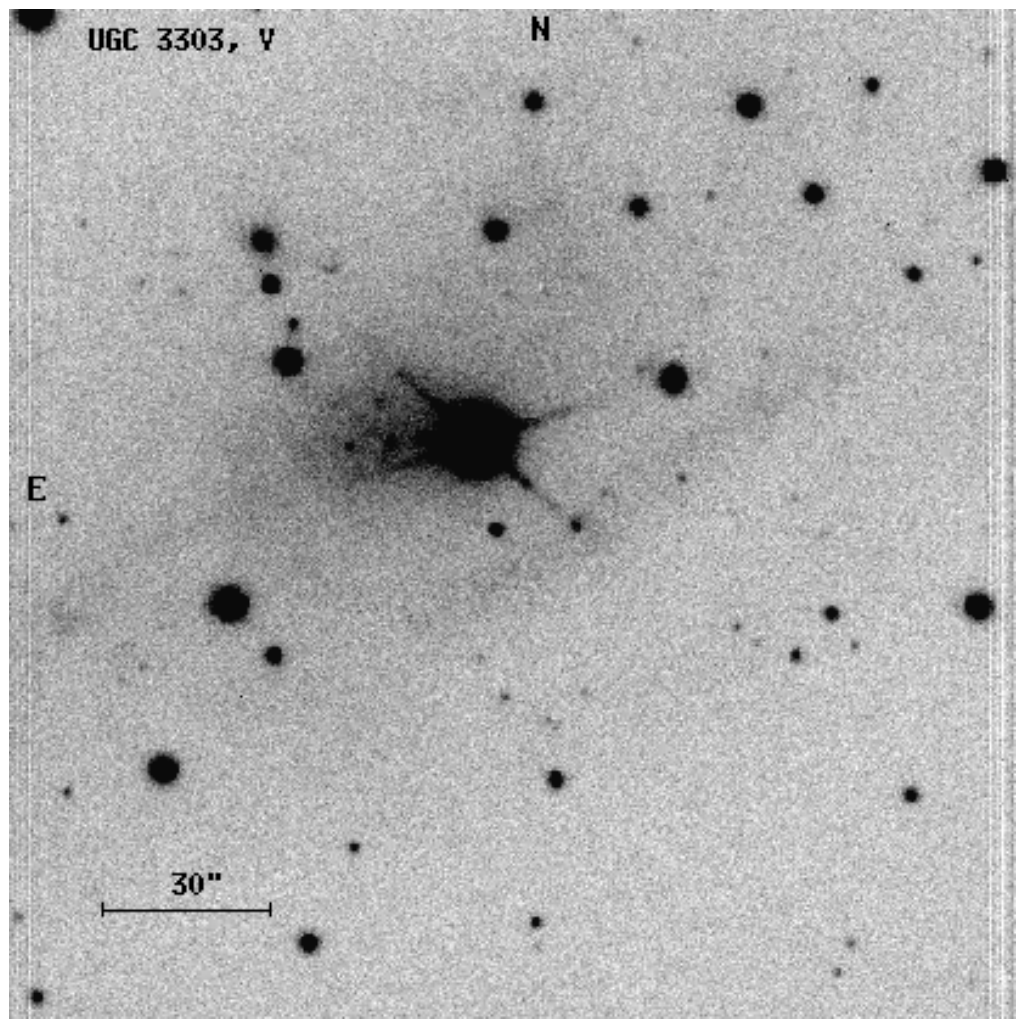

Fig. 3. The $V$ frame of UGC 3303

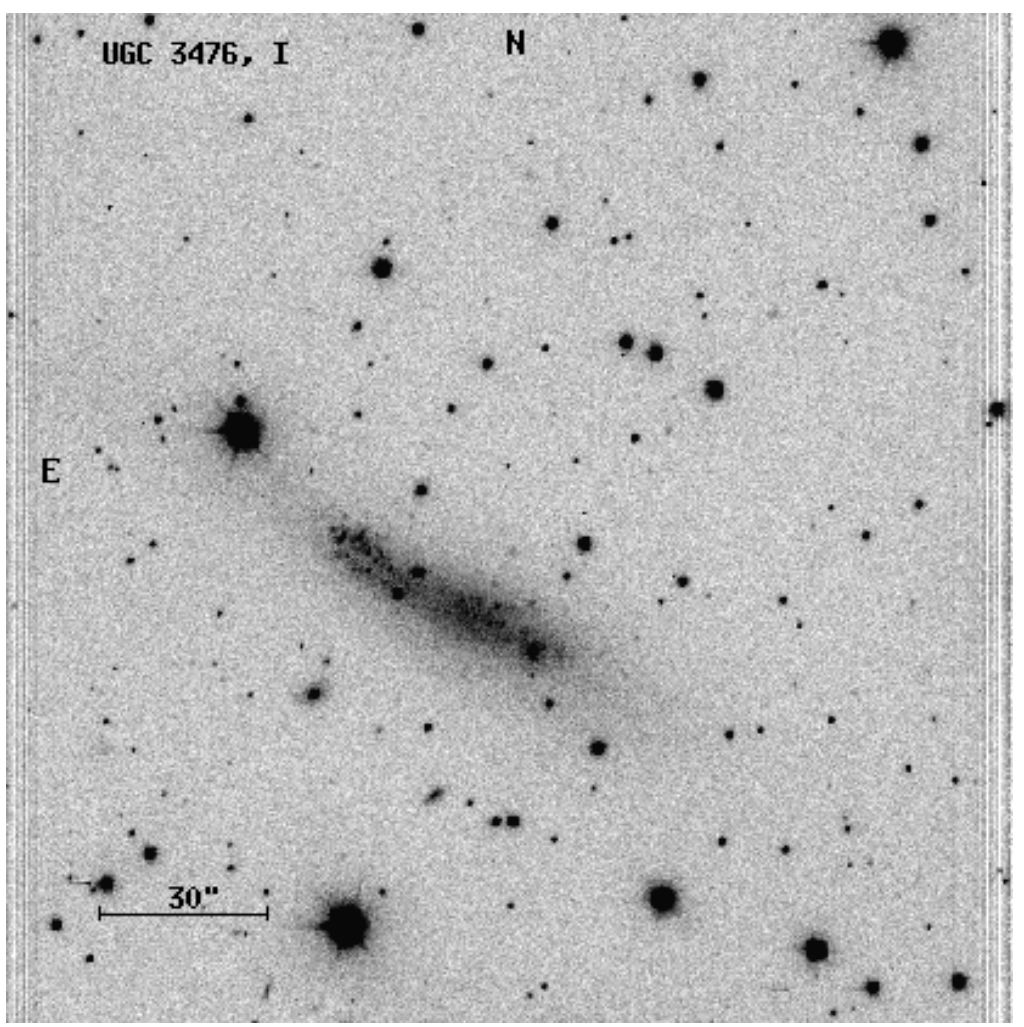

Fig. 4. The $I$ frame of UGC 3476 


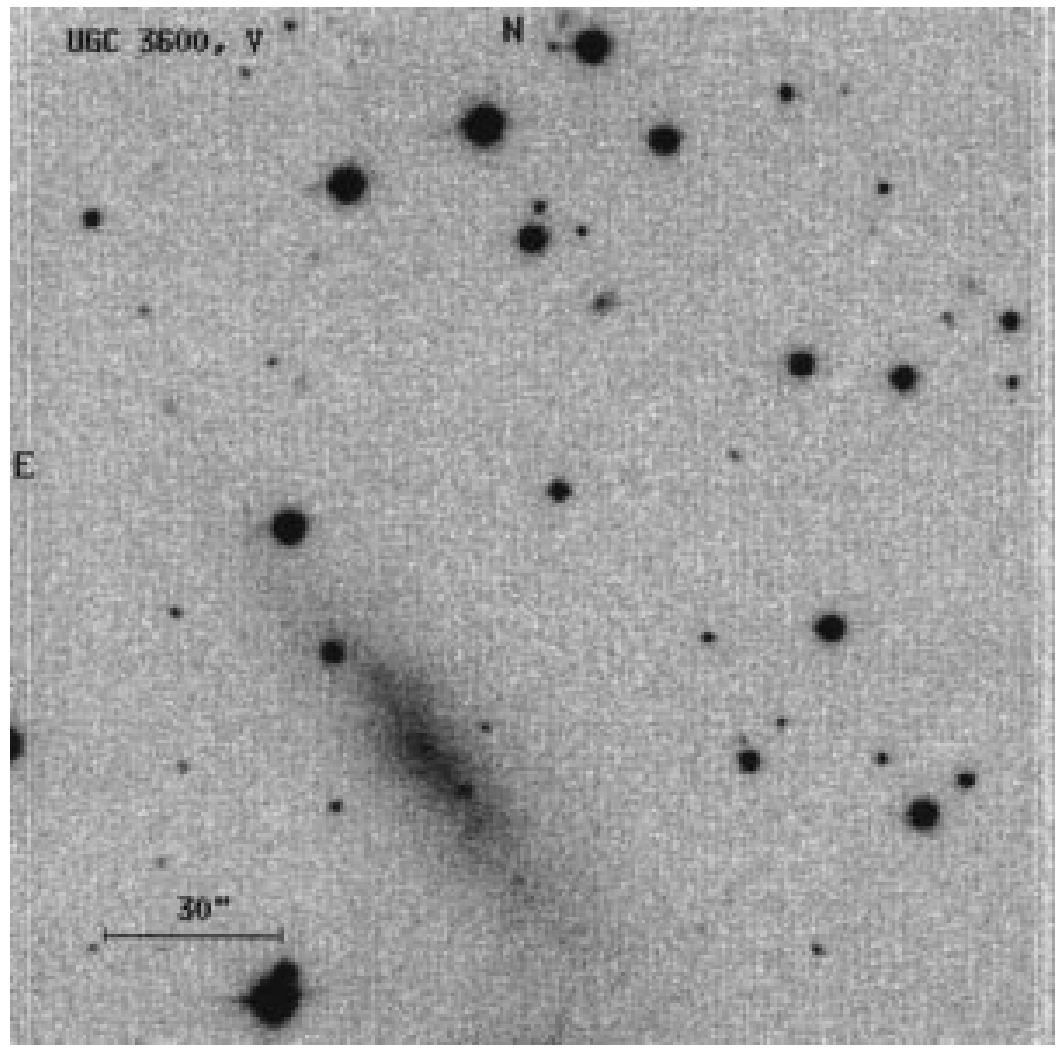

Fig. 5. The $V$ frame of UGC 3600

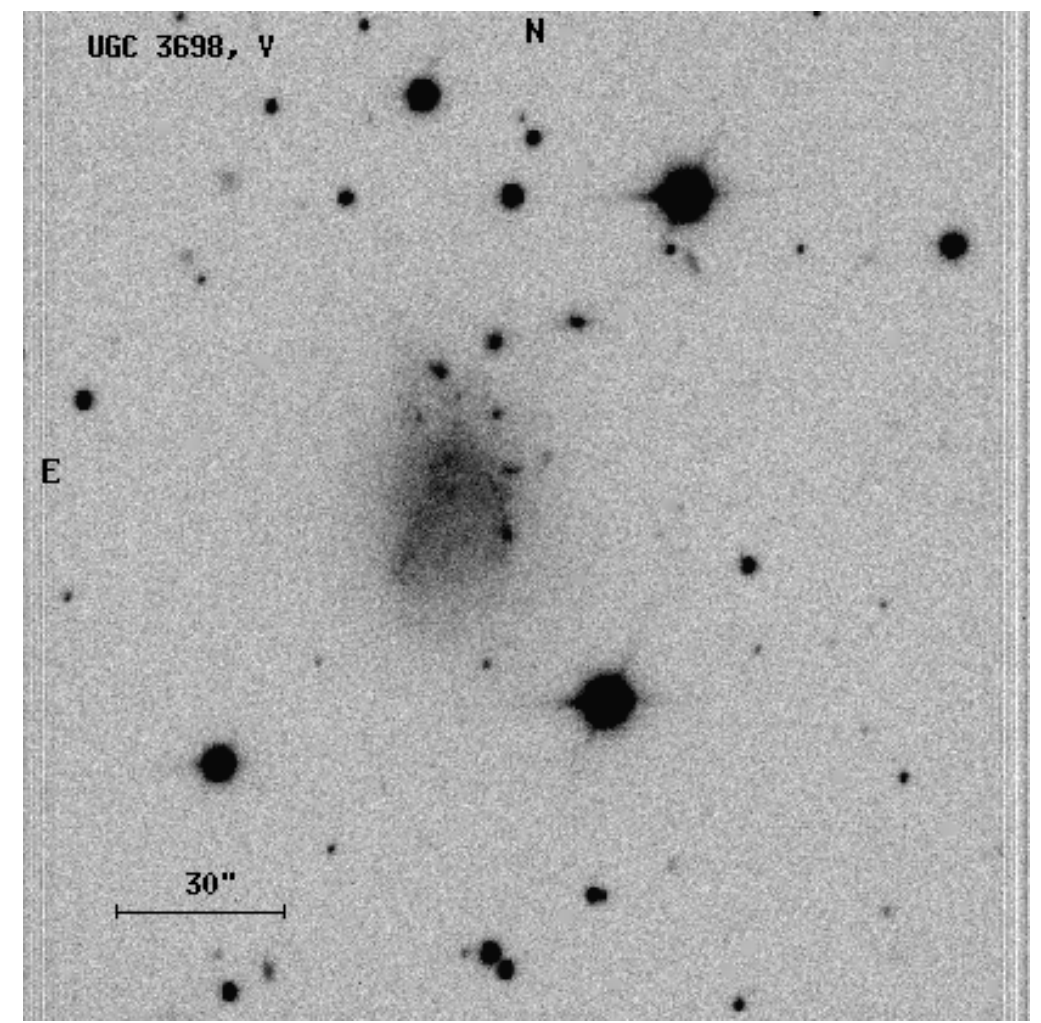

Fig. 6. The $V$ frame of UGC 3698 


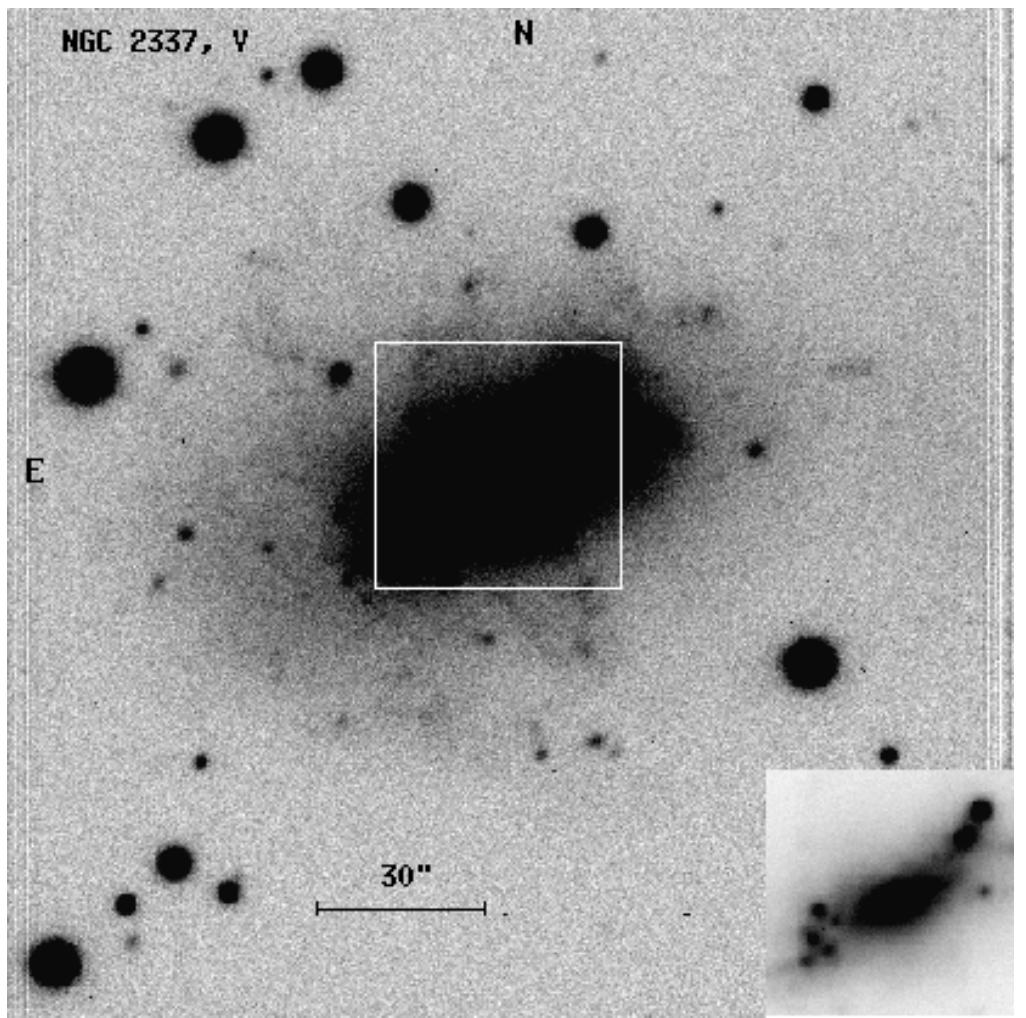

Fig. 7. The $V$ frame of NGC 2337

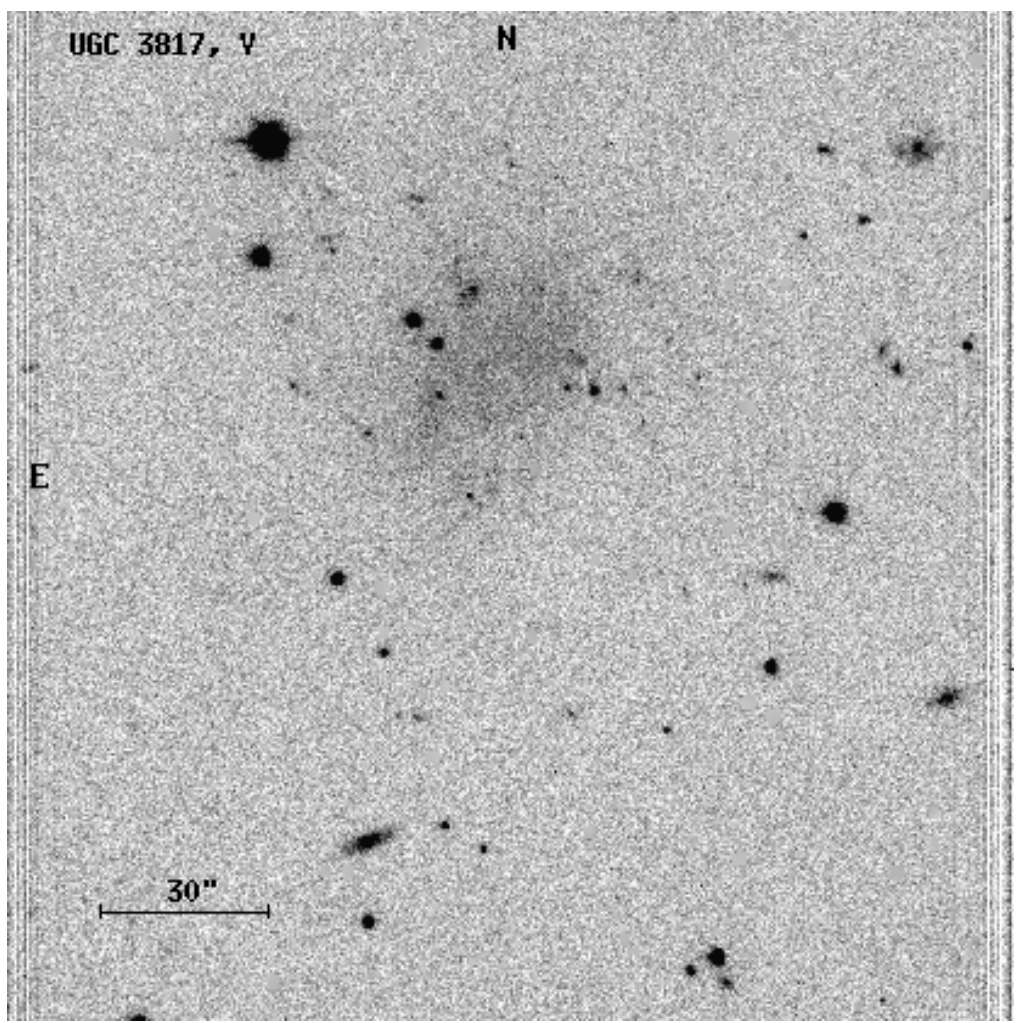

Fig. 8. The $V$ frame of UGC 3817 


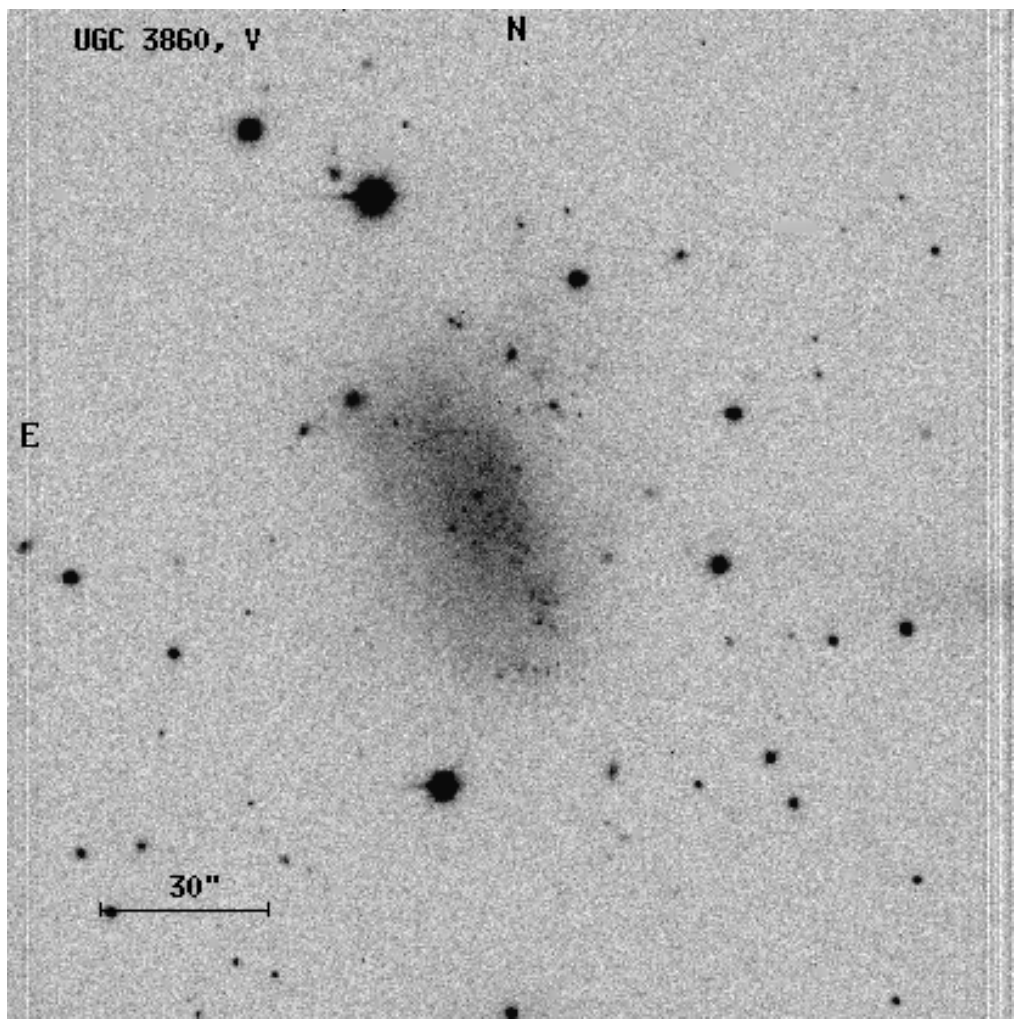

Fig. 9. The $V$ frame of UGC 3860

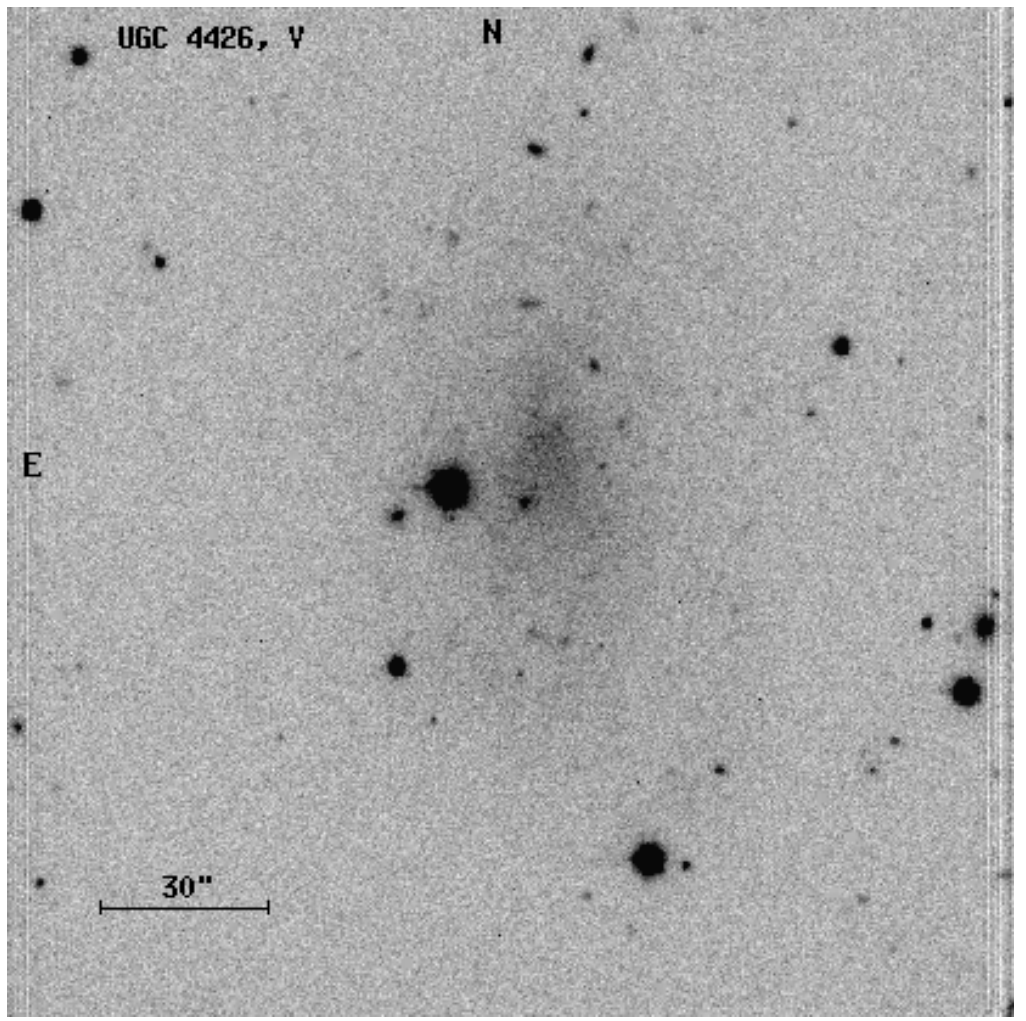

Fig. 10. The $V$ frame of UGC 4426 


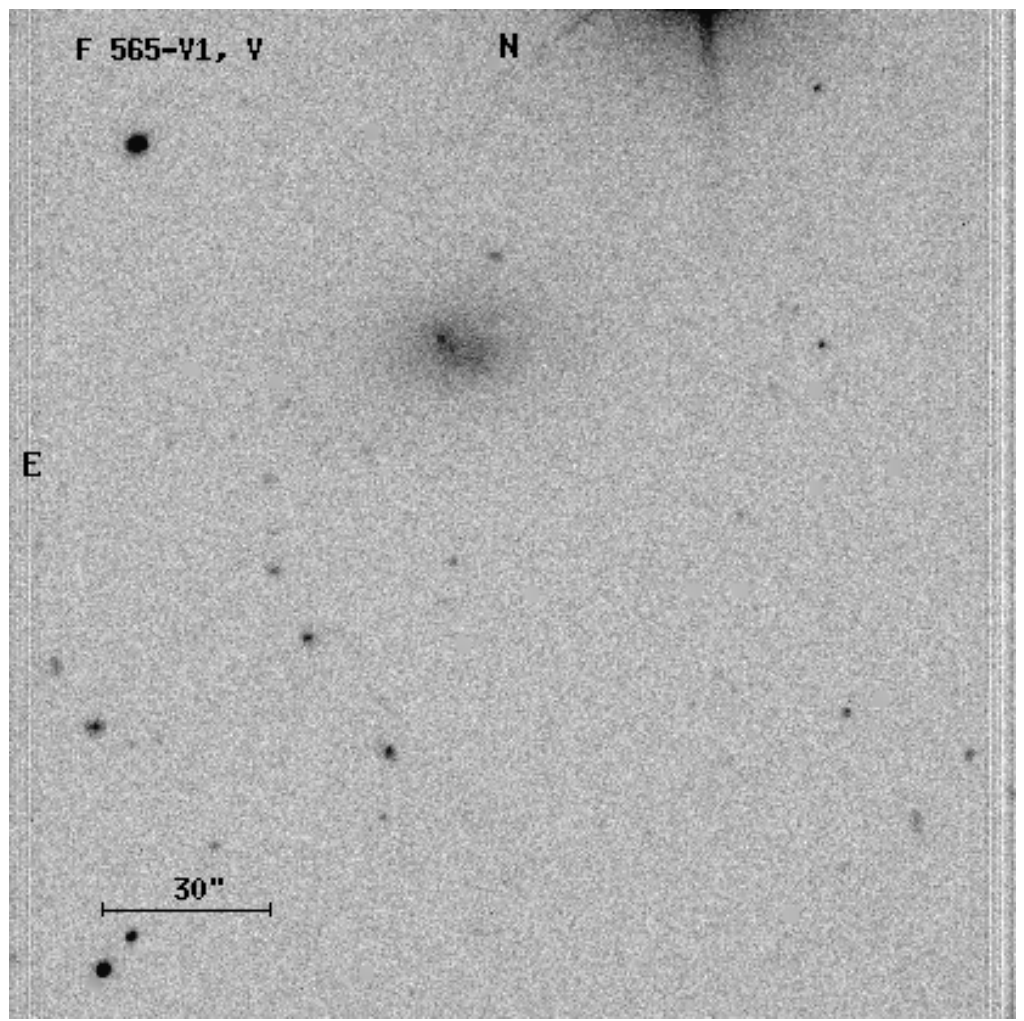

Fig. 11. The $V$ frame of F 565-v1

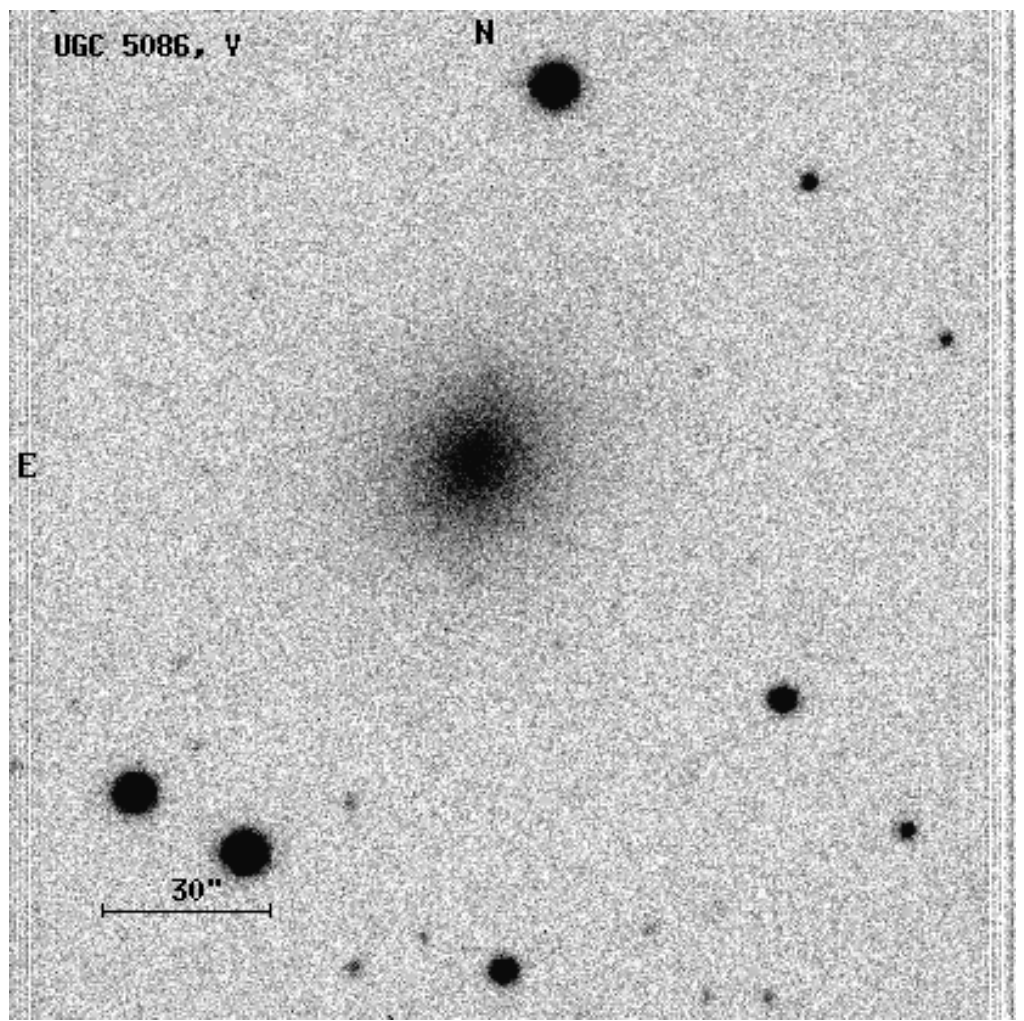

Fig. 12. The $V$ frame of UGC 5086 


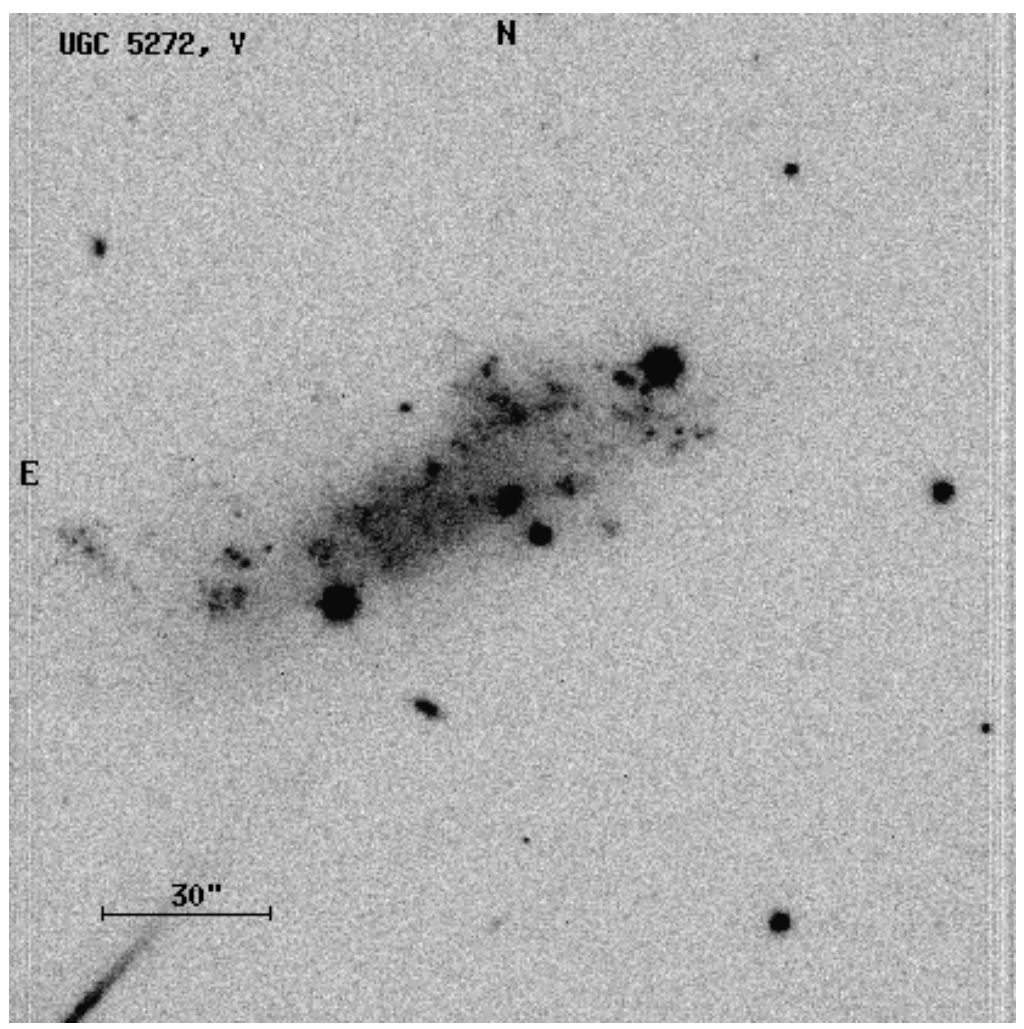

Fig. 13. The $V$ frame of UGC 5272

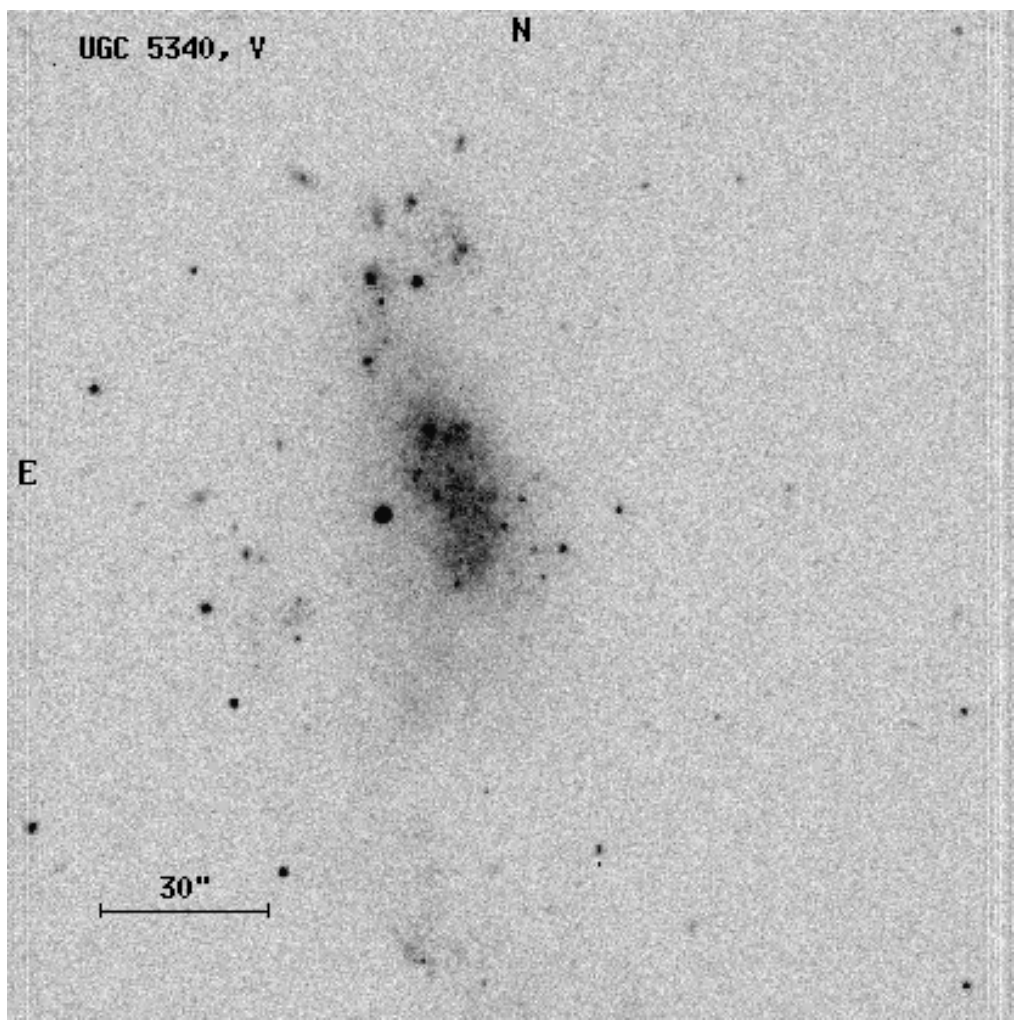

Fig. 14. The $V$ frame of UGC 5340 


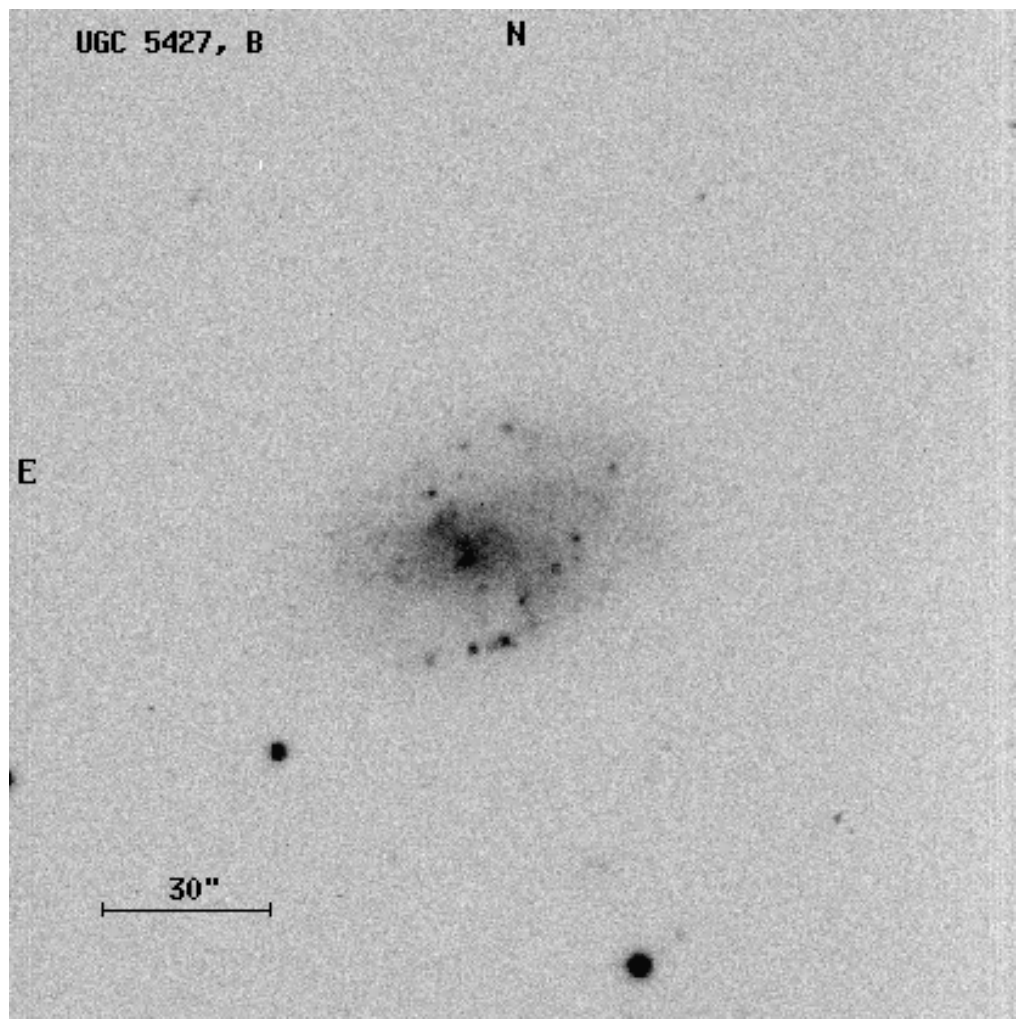

Fig. 15. The $B$ frame of UGC 5427

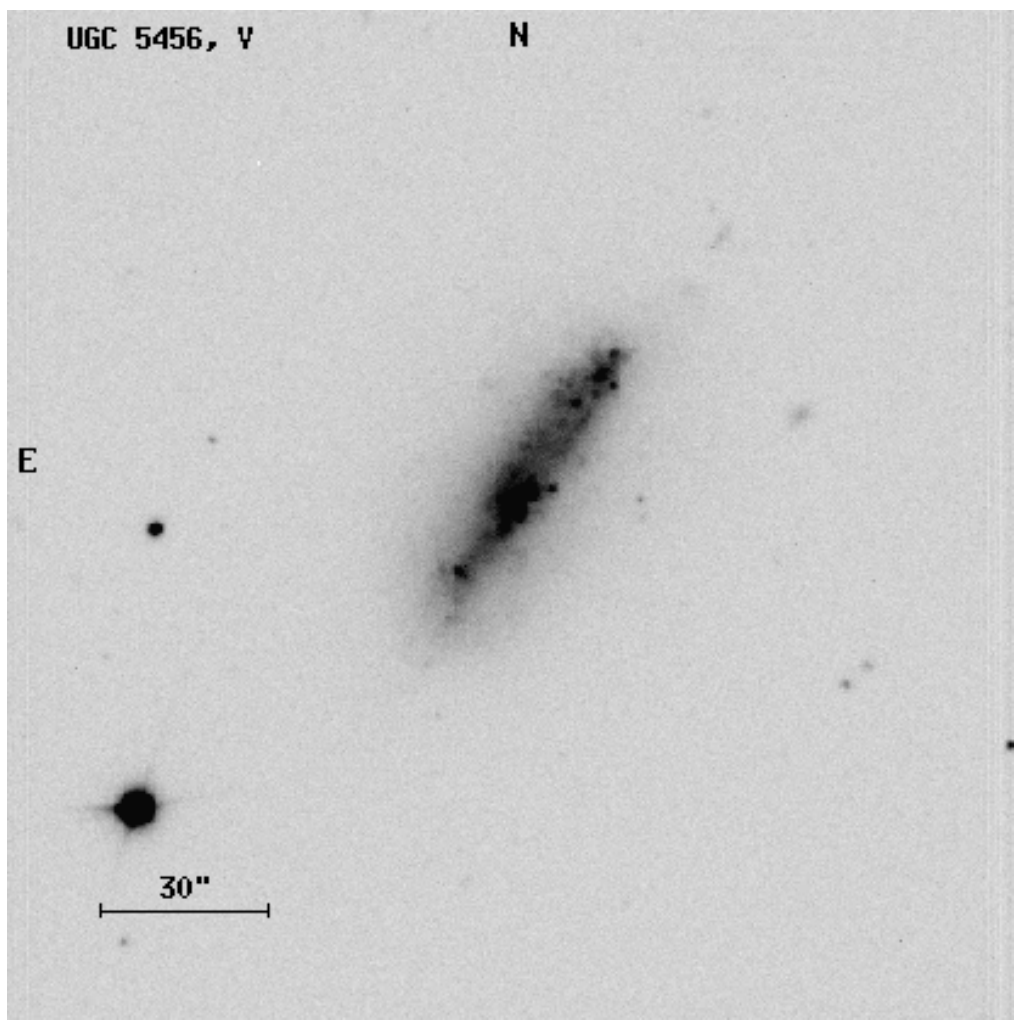

Fig. 16. The $V$ frame of UGC 5456 


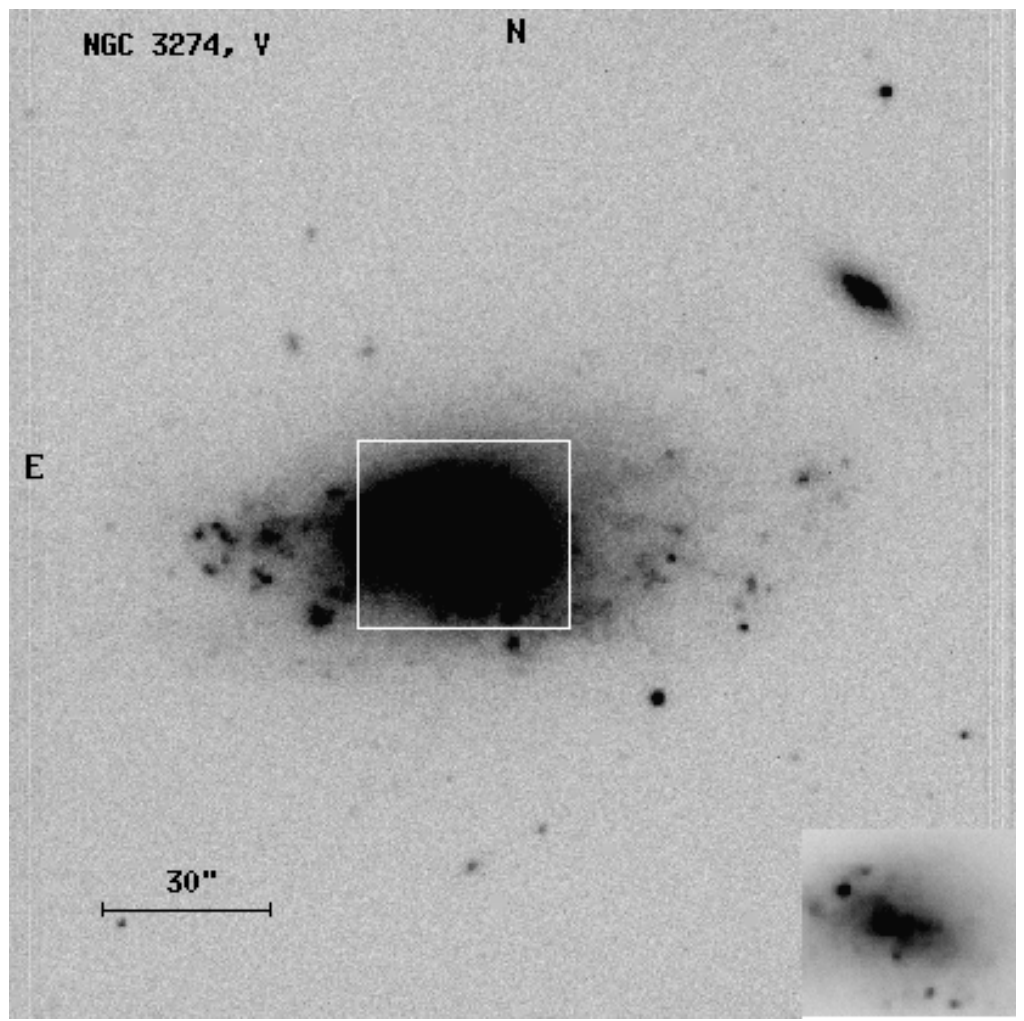

Fig. 17. The $V$ frame of NGC 3274

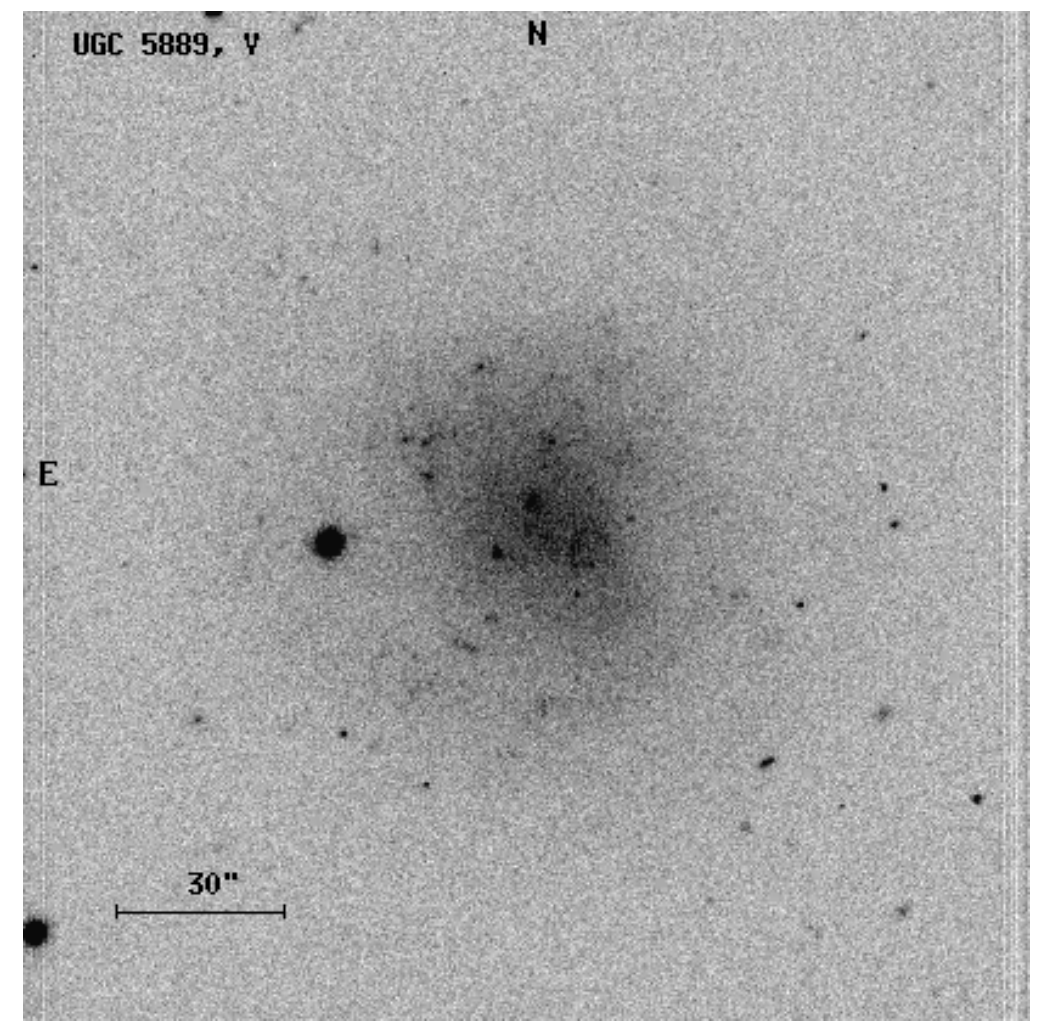

Fig. 18. The $V$ frame of UGC 5889 


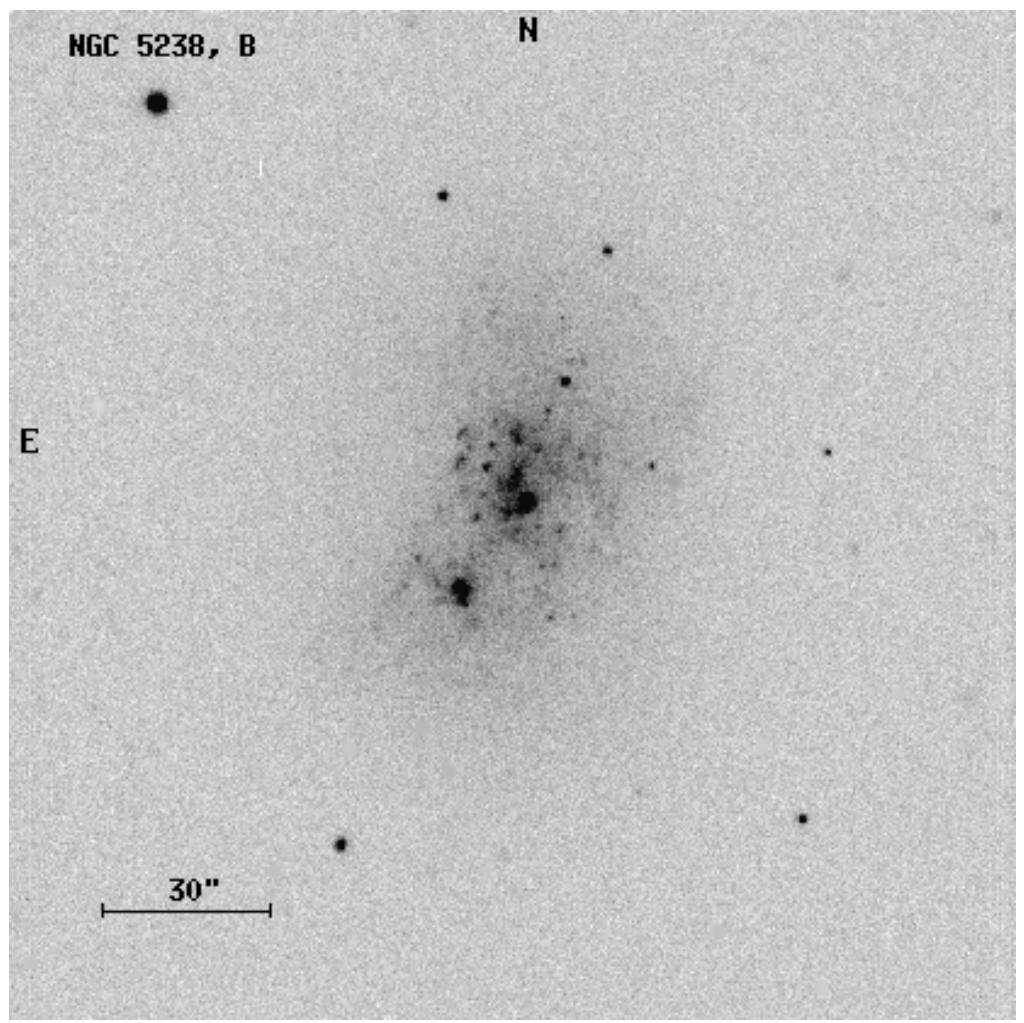

Fig. 19. The $B$ frame of NGC 5238

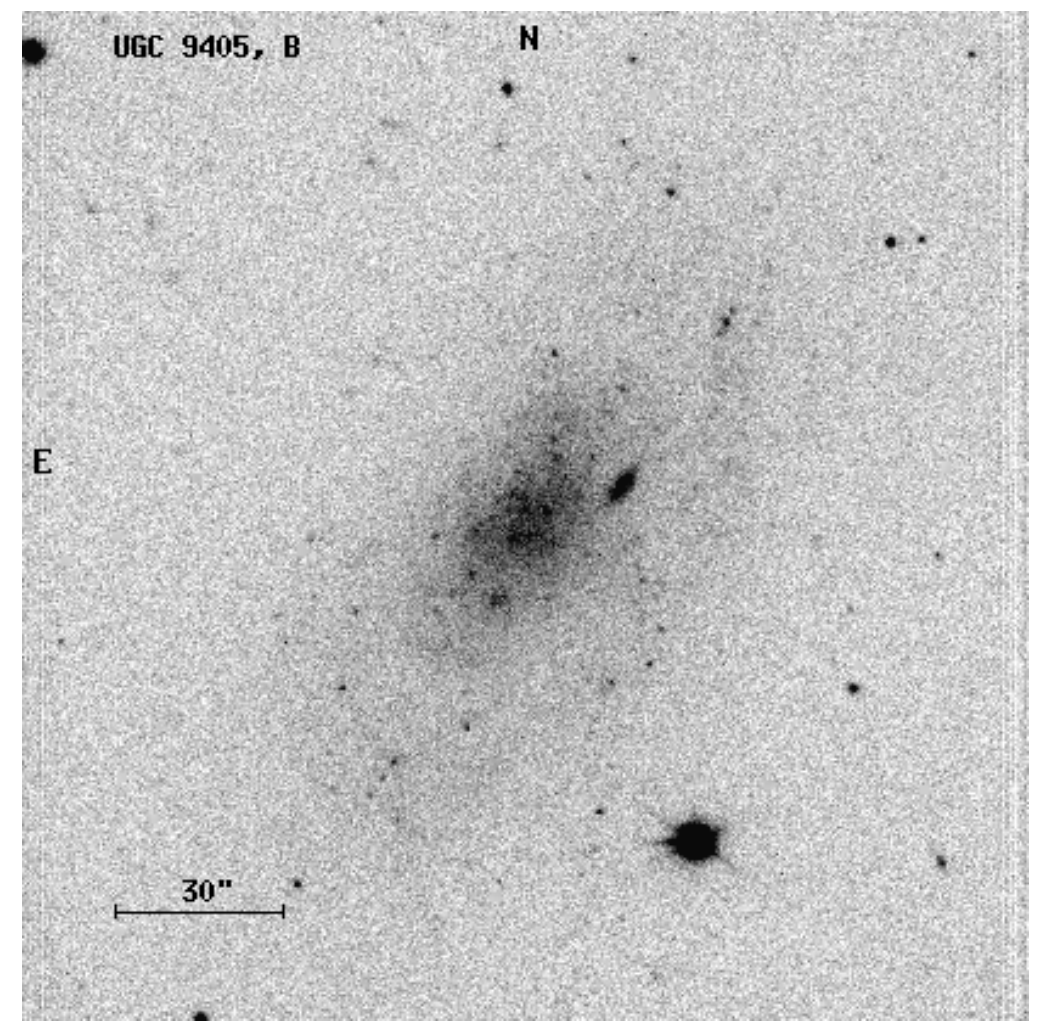

Fig. 20. The $B$ frame of UGC 9405 
UGC $4426=$ DDO 52. This is a low surface brightness galaxy with a hint of a diffuse spiral structure. It does not contain blue stellar complexes, except for object \#21 with $V=21.21, B-V=-0.07$ near the galaxy centre. The three brightest blue stars (\#15, 29 and 24 in Table 9) yield a distance modulus $\mu_{0}(3 B)=30.62$, which seems overestimated due to the apparent absence of other young massive stars in UGC 4426. As a more realistic estimate of the galaxy distance we give $\mu_{0}(R 1)=28.76$, derived from the red star \#10 with $V=21.26$ and $B-V=+1.63$.

F 565-v1. This tiny system was found by Schombert \& Bothun (1988) in a search for low surface brightness galaxies. Schombert et al. (1992) detected it in the HI $21 \mathrm{~cm}$ line. The galaxy central part shows its irregular structure (Fig. 11), however, the galaxy itself seems to be unresolved into stars down to $B_{\lim }=22.5 \mathrm{mag}$, which yields as a rough lower limit $\mu_{0}(3 B)>29.5$.

UGC 5086 = F 565-v3. This galaxy of low surface brightness is separated by 8 arcmin from the nearby giant spiral NGC 2903. Thuan \& Seitzer (1979) ascribe to it a radial velocity of $+543 \mathrm{~km} \mathrm{~s}^{-1}$. However, this detection may be spurious, due to the confusion with strong HI flux from NGC 2903, because the HI flux and linewidth presented by them for NGC 5086 are not consistent with its luminosity and structure. According to our data the galaxy looks unresolved into stars, having the regular shape and the yellow colour typical of elliptical dwarf systems. Based on the physical association of NGC 5086 with NGC 2903, we adopt for it the same distance modulus, 29.36 (Drozdowsky \& Karachentsev 1998).

UGC $5272=$ DDO $64=$ IRAS 09472+3147. Hopp \& Schulte-Ladbeck (1991) have already resolved this dwarf into stars, but have not estimated its distance. The very disturbed shape of the galaxy, the presence of many blue knots and the considerable IR flux suggest an active starburst stage. It is rather difficult to distinguish between compact blue objects and single stars in the galaxy. Adopting the starlike objects: \#38, 10 and 9 as blue supergiant candidates, we derive the galaxy distance modulus of 29.26. Within the galaxy boundary there are no red stars above our photometric limit.

UGC $5340=$ DDO $68=$ VV 542. Among other irregular dwarfs this galaxy stands out for its disturbed structure. It is listed by Vorontsov-Velyaminov (1977) in the Atlas of interacting galaxies as \#542. However, in the wide surroundings of UGC 5340 there are no other galaxies which could be responsible for the strong tidal disturbance of this system.

UGC 5340 has been resolved into stars for the first time by Hopp \& Schulte-Ladbeck (1995). The results of our photometry of the brightest stars are presented in Table 11 and as a CM diagram (Fig. 22). The galaxy is mainly populated by blue stars. Three brightest ones, \#38, 49, and 30 with $\langle B(3 B)\rangle=21.32$ yield a distance modulus of 28.85 . Here we have ignored the brightest object \#41 with $V=19.37$ and $B-V=0.03$, whose luminosity exceeds that of others by more than two magnitudes. This object may be an unresolved multiple system of young stars. Among two red stars with $B-V>+1.6$ the brighter one belongs apparently to the foreground, and the second (\#10) with $V=21.70$ and $B-V=+1.71$ yields a distance modulus $\mu_{0}(R 1)=29.39$.

It should be noted that UGC 5340 has an unusually blue integral colour, $(B-V)_{\mathrm{T}}=0.18$, which agrees well with the colour $(B-R)_{\mathrm{T}}=0.32$ according to Hopp \& Schulte-Ladbeck (1995). This feature, as well as the high hydrogen mass-to-luminosity ratio, $M(\mathrm{HI}) / L_{B}>$ $1 M_{\odot} / L_{\odot}$, may indicate a young galaxy. It seems reasonable to study this system in more detail for investigating its velocity field as well as the abundance of heavy elements.

UGC 5427. With its brightness gradient and its chain of knots resembling a spiral arm, this dwarf galaxy may easily be confused with a distant spiral one. Via the three brightest blue starlike objects we have estimated its distance modulus as 29.26 .

UGC 5456 = IRAS 10046+1036. As an IRAS source, this isolated knotty galaxy of high surface brightness contains a considerable amount of dust. Its blue stars are clustered in nests. In such a crowded field the image parameter SHARP does not guarantee reliable separation of starlike objects from nonstellar ones. Via three brightest blue objects (\#35, 13 and 21 in Table 13) we derived a distance modulus of 27.19. Here we disregard the brightest object \#10 with $V=19.15$ and $B-V=0.14$, situated in the bright northern nest. The distance $D=2.74 \mathrm{Mpc}$ seems to be in disagreement with the radial velocity of the galaxy, $V_{0}=+368 \mathrm{~km} \mathrm{~s}^{-1}$, as well with the rate of its resolution into stars. Being at a stage of active star formation, this nearby galaxy would be a suitable target for a detail study with the Hubble Space Telescope.

NGC $3274=$ UGC 5721 = IRAS 10294+2755. The galaxy has a considerable radial brightness gradient and many nonstellar bluish knots. Its central region is shown on the insert of Fig. 17. Three brightest blue supergiant candidates, \#34, 40, and 35 in Table 14, give us a distance modulus of 29.08 , which is lower than the estimate $\mu_{0}(3 B)=29.51$ derived by Georgiev et al. (1997). We have not found red stars in this galaxy.

UGC $5889=$ NGC 3377A $=$ DDO 88. This is a diffuse irregular galaxy with a red colour $(B-V)_{\mathrm{T}}=0.60$. All blue stars in it are fainter than $B=21.5$. The three brightest of them yield a distance modulus of 29.85 .

NGC $5238=$ UGC 8565 = CPG $384=$ VV $828=$ I Zw 64 $=$ Mkn 1479. As a blue compact object with strong $\mathrm{H}_{\alpha}$ emission, NGC 5238 is in the lists of Zwicky (1971) and Markarian (1967). Karachentsev (1972) and VorontsovVelyaminov (1977) classified it as an interacting double system. According to Karachentsev (1996), this dwarf galaxy, resembling an intergalactic HII region, belongs to the outskirts of the group around M 101. The mean colour index of NGC 5238 increases smoothly from 0.27 in the 


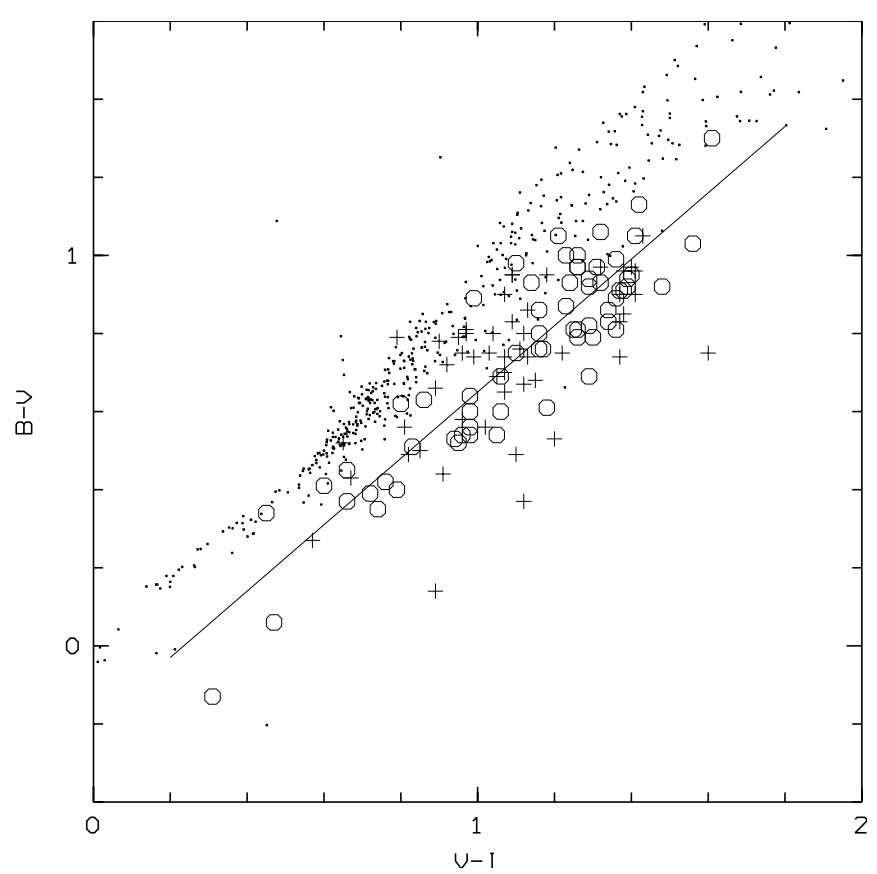

Fig. 21. $(B-V)$ vs. $(V-I)$ diagram for the standard stars (dots), and galaxies (open circles and crosses)

centre to $\left\langle B-V>_{\mathrm{T}}=0.40\right.$ within the faintest isophote, which points out to the presence of an older stellar population in the galaxy. Via the brightest three blue stars the galaxy distance modulus is 28.57 , exactly the same as the previous estimate by Karachentsev et al. (1994). In determining the distance, we have not taken into account the central bright starlike object with $V=18.27$ and $B-V=-0.44$, which may be the galaxy nucleus or a blue compact association.

UGC $9405=$ DDO 194. Like the previous galaxy, this diffuse dwarf system of regular shape is a probable outer member of the group around the giant spiral M 101. A distant elliptical galaxy is projected at the West of its central part. In the outer regions of UGC 9405 one suspects a very faint spiral arm. Such a feature is seldom met in dwarf galaxies. The central part of UGC 9405 has been resolved into stars by Karachentsev et al. (1994), who have made a rough distance modulus estimate, $\mu_{0}(R 1)=29.4(:)$.

The results of our photometry for 67 brightest stars are presented as a CM diagram (Fig. 23). The stars situated outside the visible boundary of the galaxy are indicated by crosses. From the three brightest blue supergiant candidates with $\langle B(3 B)\rangle=22.00$ the galaxy distance modulus is 29.89. Identifying the star \#24 in Table 17 with a red supergiant, we derive another estimate, $\mu_{0}(R 1)=29.16$. The true distance of the galaxy is rather uncertain; we adopt for it the average of the two determinations above.

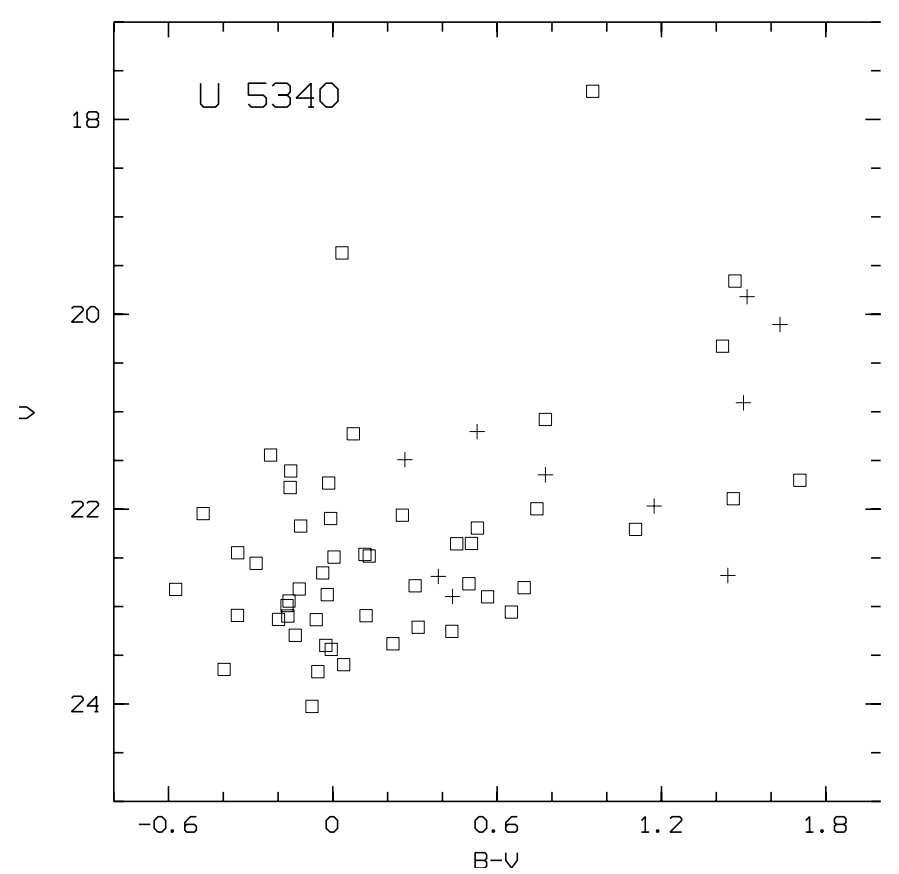

Fig. 22. $V$ vs. $(B-V)$ diagram for UGC 5340. Open squares correspond to stars measured within the galaxy boundary and crosses indicate the foreground stars

\section{Conclusions}

Taking advantage of the high quality of the Nordic telescope optics, we carried out a detailed two-colour imaging of 20 irregular galaxies. Most of them were resolved into stars for the first time. We made photometry of the brightest stellar population and also measured the integral magnitudes and colour indices of the galaxies. Based on the magnitudes of the brightest blue stars we estimated distances to the galaxies, which lie in the interval of $[5.1-9.4] \mathrm{Mpc}$.

Besides UGC 3698 and UGC 5086, the studied galaxies are well isolated systems, whose crossing time with respect to their nearest massive neighbours is of the order of the cosmological time, $\sim 10^{10}$ years. Evolution of such isolated dwarf galaxies should not be affected by tidal disturbances. Nevertheless, these objects show impressively diversified morphologies, which are likely to result from their starburst activity.

The absolute magnitudes of the galaxies lie in the interval $[-12.5,-16.9]$ with a median of -14.6 , and their integral colours, $(B-V)_{\mathrm{T}}^{0}$, are concentrated within $[0.30,0.70]$ with a median of 0.47 . Outside this colour index interval there is only one galaxy, UGC 5340, with $(B-V)_{\mathrm{T}}^{0}=0.17$. It has also a high $M(\mathrm{HI}) / L_{B}$ ratio, being probably a young system.

Note that among these 20 nearby irregular dwarfs only 4 objects are IRAS sources. If we rank them according to their apparent magnitude, they occupy positions \#3-6 in our sample, whereas their classification by the mean surface brightness give them positions \#2-5. Therefore, 


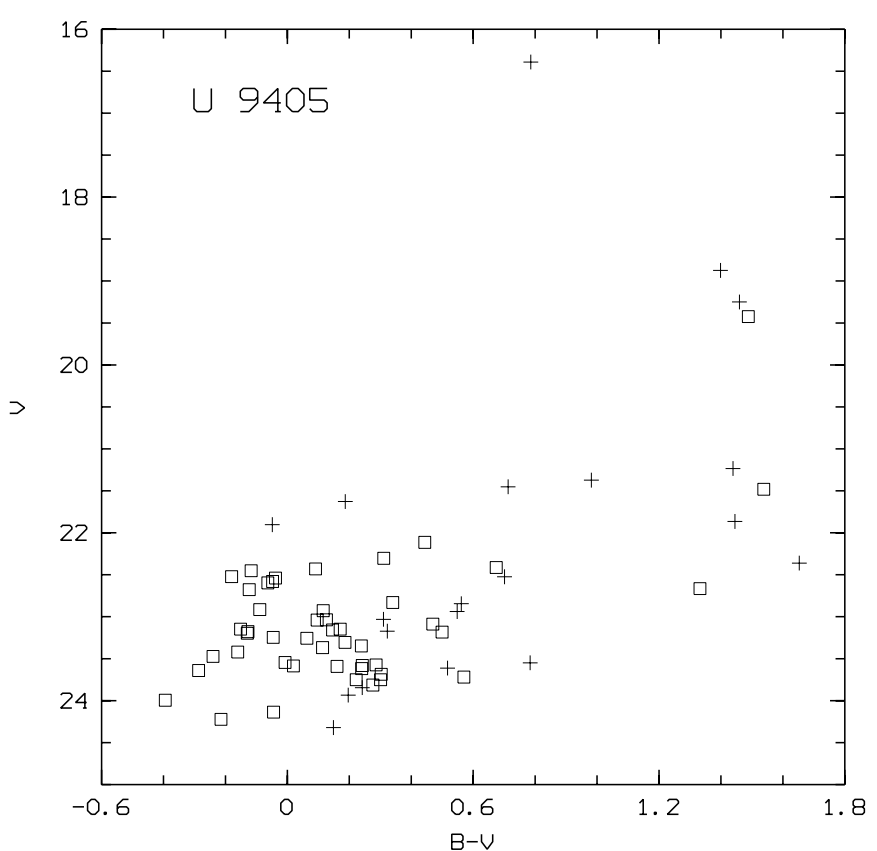

Fig. 23. $V$ vs. $(B-V)$ diagram for UGC 9405. Crosses indicate the foreground stars

the IR-flux from dwarf galaxies seems to exhibit a correlation both with their optical flux and with their surface brightness. Besides, the dwarf galaxies detected in the IR have a slightly bluer integral colour.

Because the radial velocities of the considered galaxies can not be much affected by neighbours, one can use them to estimate the local value of the Hubble parameter, $H=V_{0} / D$. According to the data of Table 18, individual estimates of $\mathrm{H}$ have a median of $65 \mathrm{~km} \mathrm{~s}^{-1} / \mathrm{Mpc}$. This value is in reasonable agreement with other estimates of the Hubble parameter.

Acknowledgements. We are indebted to Leo Takalo and Pekka Heinamaki, who took part in the observations, and Serafim Kajsin, who made preliminary processing of the data. This work is partly supported by INTAS-RFBR grant No. 95-1390.

\section{References}

de Vaucouleurs G., 1978, ApJ 224, 14; 224, 710

Drozdovsky I.O., Karachentsev I.D., 1998, A\&AS (in preparation)

Fukugita M., Shimasaku K., Ichikawa T., 1995, PASP 107, 945

Georgiev Ts.B., Karachentsev I.D., Tikhonov N.A., 1997, Astron. Lett. 23, 514

Heraudeau Ph., Simien F., 1996, A\&AS 118, 111

Hopp U., Schulte-Ladbeck R.E., 1991, A\&A 248, 1

Hopp U., Schulte-Ladbeck R.E., 1995, A\&AS 111, 527

Karachentseva V.E., 1973, Communic. Spec. Astrophys. Obs. 8,3

Karachentsev I.D., 1972, Communic. Spec. Astrophys. Obs. 7, 3

Karachentsev I.D., 1996, A\&A 305, 33

Karachentsev I.D., Drozdovsky I.O., 1998, A\&AS (accepted)

Karachentsev I.D., Drozdovsky I.O., Kajsin S., Takalo L., Heinamaki P., Valtonen M., 1997, A\&AS 124, 559

Karachentsev I.D., Karachentseva V.E., Parnovsky S.L., 1993, Astron. Nachr. 314, 97

Karachentsev I.D., Kopylov A.I., Kopylova F.G., 1994, Bull. Spec. Asrtophys. Obs. 38, 5

Karachentsev I.D., Makarov D.I., 1996, AJ 111, 794

Karachentsev I., Musella I., 1996, A\&A 315, 348

Karachentsev I.D., Tikhonov N.A., 1994, A\&A 286, 718

Landolt A.U., 1992, AJ 104, 340

Makarova L., Karachentsev I., Takalo L., Heinamaki P., Valtonen M., 1998, A\&AS 128, 459

Markarian B.E., 1967, Astrofizika 3, 55

Paturel G., Bottinelli L., Fouque P., Gouguenheim L., 1992, Catalogue of Principal Galaxies: PGC, Observatoire de Lyon

Reshetnikov V., Combes F., 1996, A\&AS 116, 417

Sandage A., Tammann G.A., 1974, ApJ 194, 223; 194, 559

Schombert J.M., Bothun G.D., 1988, AJ 95, 1389

Schombert J.M., Bothun G.D., Schneider S.E., McGaugh S.S., 1992, AJ 103, 1107

Stetson P., 1987, PASP 99, 191

Thuan T.X., Seitzer P.O., 1979, ApJ 231, 327

Vorontsov-Velyaminov B.A., 1977, A\&AS 28, 1

Zwicky F., 1971, Catalog of Selected Compact Galaxies and Post-Eruptive Galaxies, Pasadena, 1 